\title{
Programas e intervenciones educativas ofrecidas al personal farmacéutico para mejorar la prestación de los servicios asistenciales farmacéuticos: una revisión estructurada
}

\author{
Educational programs and interventions offered to \\ pharmacy staff to improve the delivery of pharmaceutical \\ assistance services: A structured review \\ Mauricio Ceballos $^{1}{ }^{\mathbb{D}} ;$ Juliana Madrigal-Cadavid $^{1}{ }^{\circledR} ;$ Pedro Amariles $^{1}{ }^{(1)}$
}

Forma de citar: Ceballos M, Madrigal-Cadavid J, Amariles P. Programas e intervenciones educativas ofrecidas al personal farmacéutico para mejorar la prestación de los servicios asistenciales farmacéuticos: una revisión estructurada. Salud UIS. 53: e21010. doi: https://doi.org/10.18273/saluduis.53.e:21010 @ () ()

\section{Resumen}

Objetivo: describir los programas y las intervenciones educativas ofrecidas al personal farmacéutico, para mejorar la prestación de los servicios asistenciales farmacéuticos y los métodos utilizados para evaluar la efectividad de estos programas. Métodos: se realizó una revisión estructurada, se utilizó la base de datos PubMed/Medline entre 2007 y 2019, sobre el desarrollo, la descripción, la evaluación del impacto, la efectividad o la comparación de una intervención educativa, un entrenamiento específico o un programa educativo dirigido al personal farmacéutico de farmacias comunitarias (ambulatorias) y/o hospitalarias. Resultados: en total, 1290 referencias bibliográficas fueron identificadas: se incluyeron 26 referencias adicionales y se seleccionaron 90 artículos para su revisión y análisis. Se encontró que el 70\% (63) de los estudios fueron realizados en países desarrollados, principalmente en Estados Unidos, Australia, Canadá e Inglaterra. El 67\% (60) de los estudios se realizaron a nivel ambulatorio, el 10\% (9) a nivel hospitalario y el 23\% (21), en ambos contextos. Las intervenciones fueron clasificadas en tres categorías: Entrenamientos Específicos (41\%), programas de Educación Continua (43\%) y de Desarrollo Profesional Continuo, $(16 \%)$. De las metodologías o métodos de provisión de la intervención, la más común fue la presencial en el $72 \%(65)$ de los estudios, seguido de metodologías apoyadas con herramientas virtuales en el $28 \%$ (25) y en el $11 \%$ (10) de los estudios, combinaron métodos presenciales y virtuales. El tiempo de duración

1. Universidad de Antioquia, Medellín, Colombia.

Correspondencia: Mauricio Ceballos. Dirección: Calle 67 53-108, Medellín. Teléfono: +57 (4) 2198332 Correo electrónico: javier.ceballos@udea.edu.co 
de las intervenciones educativas varió entre menos de 1 hora y 120 horas. Conclusión: se describen los programas y las intervenciones educativas ofrecidas al personal farmacéutico, principalmente para mejorar sus competencias laborales y la prestación de los servicios asistenciales farmacéuticos, además de los métodos utilizados para evaluar la efectividad de las intervenciones educativas.

Palabras clave: Personal Farmacéutico; Farmacéuticos; Educación Continua; Desarrollo Profesional Continuo; Farmacias.

\begin{abstract}
Objective: To describe the educational programs and interventions offered to pharmacy staff, to improve the delivery of pharmaceutical healthcare services and the methods used to evaluate the programs' effectiveness. Methods: A structured review was performed using the PubMed/Medline database from 2007 to 2019. Results: In total, 1290 bibliographic references were identified; besides, 26 references identified by other sources were included and 90 articles for review and analysis were selected. It was found that $70 \%(63)$ of the studies were made in developed countries, mainly in the United States, Australia, Canada, and England. 67\% (60) of the studies were carried out at the outpatient level, $10 \%(9)$ at the hospital level, and 23\% (21) in both contexts. The interventions were classified into three categories: Specific settings (41\%), Continuing Education (43\%), and Continuing Professional Development $(16 \%)$ programs. The most common methodology or delivery methods for the intervention were face-to-face in $72 \%$ (65) of the studies, followed by methodologies supported by virtual tools in $28 \%(25)$ and $11 \%$ (10) of the studies combined face-to-face and virtual methods. The duration of the educational interventions ranged from less than 1 hour to 120 hours. Conclusion: The educational programs and interventions offered to the pharmacy staff are described, mainly to improve their labor competencies. Additionally the provision of pharmaceutical healthcare services and the methods used to evaluate the effectiveness of the educational intervention.
\end{abstract}

Keywords: Pharmacy Staff; Pharmacists; Pharmacies; Continuing Education; Continuing Professional Development.

\section{Introducción}

En la actualidad existen desafíos y oportunidades para el personal farmacéutico (profesionales, tecnólogos y auxiliares/técnicos) y en particular, aquellos que laboran en los servicios farmacéuticos ambulatorios (para Colombia, similar al concepto de farmacia comunitaria) y las droguerías y farmacias-droguerías (establecimientos farmacéuticos minoristas 0 ambulatorios), ya que están entre las principales y más accesibles fuentes de información; además, son lugares desde los cuales se puede ayudar a los pacientes en diferentes condiciones de salud ${ }^{1}$. Desde hace algunos años, la Organización Mundial de la Salud (OMS) y la Federación Internacional Farmacéutica (FIP) han impulsado su evolución hacia una farmacia más orientada y centrada en el paciente ${ }^{2}$, aunque a nivel comunitario o ambulatorio sus actividades principales son más administrativas. En este contexto, la dispensación de medicamentos se limita a la simple entrega o despacho de los medicamentos. Además, existen fallas en la vigilancia y control de los procesos; por ejemplo, es común que muchos medicamentos que requieren prescripción médica se dispensen sin una formula médica válida, incluso cuando es obligatorio, como sucede en Colombia ${ }^{3,4} \mathrm{y}$ en otros países ${ }^{5}$.
En algunos estudios orientados a establecer la forma como aprenden los profesionales, se ha demostrado que el aprendizaje autodirigido se utiliza mucho más que los programas formales de educación continua ${ }^{6}$, y que la educación continua médica y farmacéutica tradicional por sí sola no facilita el cambio en la práctica ${ }^{7,8}$. Sin embargo, durante la práctica habitual, el personal farmacéutico puede encontrar problemas en los procesos y en los resultados con el uso de los medicamentos. Para abordar estos problemas, es necesario que actualicen continuamente sus competencias laborales. La OMS y FIP definen las competencias laborales como los conocimientos, habilidades y actitudes requeridos por el talento humano en salud en los diferentes ámbitos de la práctica para solucionar los problemas de salud de manera eficiente y efectiva ${ }^{9,10}$.

Desde principios de la década del 2000 los programas de Desarrollo Profesional Continuo (DPC) se han mencionado en el área farmacéutica asistencial, tanto en los Estados Unidos como en Gran Bretaña ${ }^{11}$. La FIP adoptó este concepto desde el 2002 y definió estos como la responsabilidad individual de un farmacéutico para el mantenimiento, desarrollo y ampliación sistemática de conocimientos, habilidades y actitudes para garantizar la competencia continua como profesional a lo largo de 
su carrera ${ }^{12}$. Por su parte, los programas de Educación Continua (EC) han existido por más tiempo que los de DPC a nivel mundial, y siguen siendo el pilar del aprendizaje posterior a la calificación en muchos lugares. Si bien, ambos aseguran que el aprendizaje se complete y se registre, los programas de EC se centran en la participación en eventos de educación o capacitación $\mathrm{y}$ en registrar las horas de educación recibidas ${ }^{12}$. Cada vez, hay más países que están implementando programas de EC, como programas obligatorios de aprendizaje permanente en un intento por mejorar los servicios asistenciales de las farmacias ${ }^{13}$.

La educación continua y la comunicación con otros profesionales de la salud son claves para optimizar los resultados de la medicación en todas las fases o procesos asociados a la utilización de los medicamentos. Aunque queda mucho por investigar sobre este tema, ahora existen más oportunidades y herramientas de capacitación y educación continua apoyadas por las Tecnologías de la Información y la Comunicación (TICs) ${ }^{14}$. La realidad del personal farmacéutico muestra la necesidad de aumentar la formación, cualificación y entrenamiento farmacéutico específico, mediante programas de educación formal y no formal, y centrados en las competencias laborales ${ }^{14}$. Por ello, se requiere identificar los programas e intervenciones que más podrían aumentar la efectividad de estos programas de capacitación. En este sentido, el objetivo de este trabajo fue describir los programas y las intervenciones educativas ofrecidas al personal farmacéutico para mejorar la prestación de los servicios asistenciales farmacéuticos y los métodos utilizados para evaluar la efectividad de estos programas.

\section{Metodología}

Se realizó una revisión estructurada en PubMed/ Medline de artículos publicados en inglés o español, con acceso a texto completo entre 2007/01/01 y 2019/01/31, sobre el desarrollo, la descripción, la evaluación del impacto, la efectividad o la comparación de una intervención educativa, un entrenamiento específico o un programa educativo dirigido al personal farmacéutico de farmacias comunitarias (ambulatorias) y/o hospitalarias. La búsqueda se realizó con los siguientes términos $\mathrm{MeSH} / \mathrm{DeCS}$ y operadores booleanos en título/abstract: "Pharmacies" OR "Community pharmacy" OR "Pharmaceutical Services" OR "Pharmacist" OR "Pharmacy Staff" AND "Education, Pharmacy, Continuing" OR "Competence development" OR "Training" OR
"Postgraduate education" OR "Recurrent education" OR "Adult learning" OR "Training and education" OR "Competence" OR "Continuing education" OR "Continuing Professional Development" OR "Life-long Learning" OR "Practical work training" OR "Health Knowledge, Attitudes, Practice".

Los criterios de inclusión fueron: presencia en el título o resumen, de información sobre el desarrollo, la descripción, la evaluación, el impacto, la efectividad (efecto) o la comparación de una intervención educativa, un entrenamiento específico o un programa educativo. Por su parte, los criterios de exclusión fueron: 1) publicaciones sin relación con los objetivos de la búsqueda y de la revisión y, 2) artículos con información limitada para caracterizar adecuadamente la intervención. Los artículos identificados fueron revisados por dos investigadores de forma pareada. Para ello, se revisaron los títulos y resúmenes de todos los artículos identificados para decidir su elegibilidad. Los artículos seleccionados se analizaron conjuntamente y, por consenso, se definió su inclusión o no.

\section{Formato recolección de información}

En Excel 2016 para Windows ${ }^{\circledR}$ se diseñó un formato para consignar y tabular los datos de los estudios, con la siguiente estructura: a) referencia bibliográfica; b) tipo de estudio; c) características de las farmacias; d) características del personal farmacéutico; e) ámbito del estudio (ambulatorio y/o hospitalario); f) características de las intervenciones educativas; g) objetivos de las intervenciones; h) problemas de salud y enfermedades intervenidos, i) servicios asistenciales farmacéuticos intervenidos, $\mathrm{j}$ ) desenlaces principales, $\mathrm{k}$ ) métodos de entrega de las intervenciones; 1) características de las mediciones y el seguimiento $\mathrm{y}, \mathrm{m}$ ) métodos y técnicas de recolección de la información.

\section{Resultados}

En total, 1290 referencias bibliográficas fueron identificadas con la estrategia de búsqueda; además, se incluyeron 26 identificadas en artículos incluidos y al final, se seleccionaron 90 artículos para su revisión y análisis. En la Figura 1 se ilustra el proceso de identificación y selección de los artículos. La mitad de los estudios fueron publicados entre 2012 y 2015, realizados principalmente en Estados Unidos (37\%), Australia (13\%), Canadá (12\%) e Inglaterra (8\%). En la Tabla 1 se describen los resultados principales de las intervenciones y programas educativos ofrecidos al personal farmacéutico. 

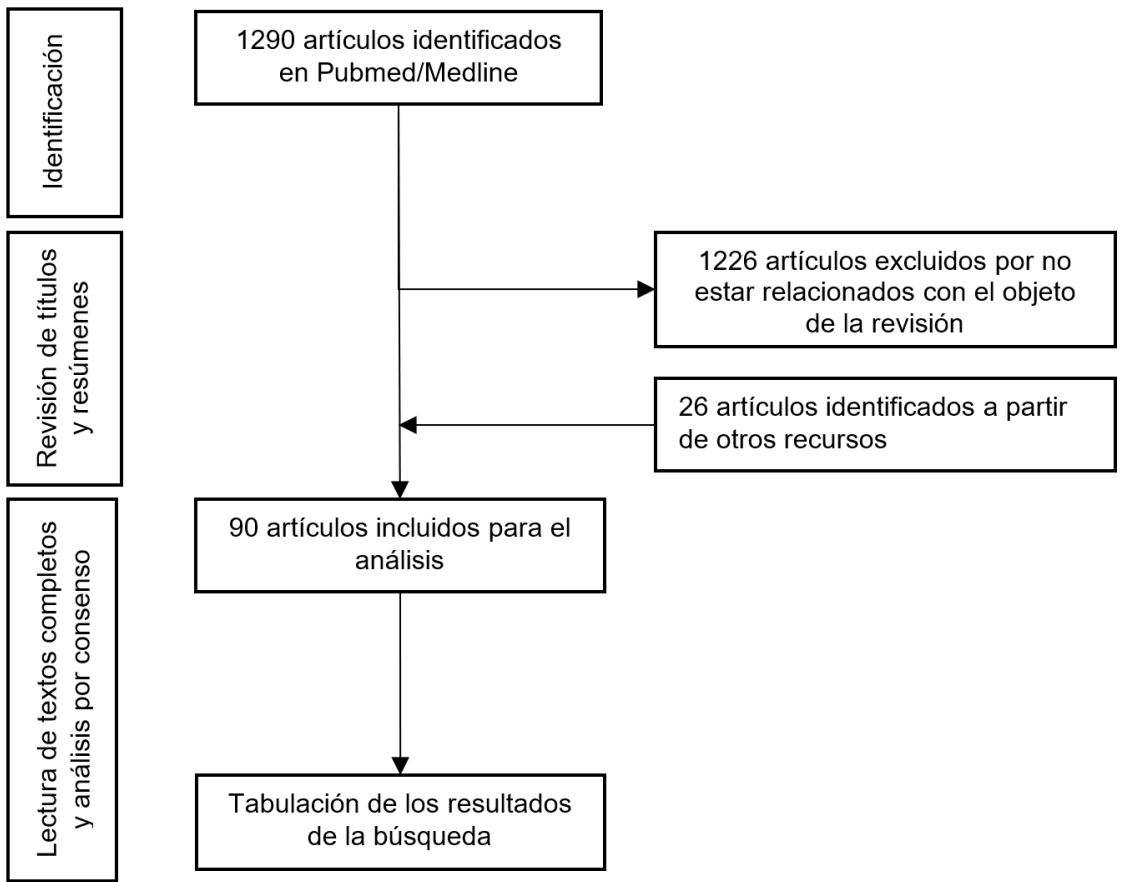

Figura 1. Identificación y selección de artículos para la revisión.

Tabla 1. Resultados principales de las intervenciones y programas educativos ofrecidos al personal farmacéutico.

\begin{tabular}{|c|c|c|c|c|c|c|c|c|c|}
\hline Autor & País & Contexto & $\begin{array}{c}\text { Nivel de } \\
\text { formación }\end{array}$ & $\begin{array}{l}\text { Diseño del } \\
\text { estudio }\end{array}$ & $\begin{array}{c}\text { Tipo } \\
\text { intervención }\end{array}$ & $\begin{array}{c}\text { Cantidad de } \\
\text { farmacias } \\
\text { incluidas }\end{array}$ & $\begin{array}{c}\text { Cantidad de } \\
\text { farmacéuticos } \\
\text { intervenidos }\end{array}$ & $\begin{array}{l}\text { Tiempo de } \\
\text { duración } \\
\text { de la } \\
\text { intervención }\end{array}$ & $\begin{array}{c}\text { Métodos } \\
\text { de la } \\
\text { intervención }\end{array}$ \\
\hline $\begin{array}{l}\text { McConnell, } \\
\text { et al. }{ }^{7}\end{array}$ & $\begin{array}{l}\text { Estados } \\
\text { Unidos }\end{array}$ & $\begin{array}{c}\text { Ambos } \\
\text { (ambulatorio } \\
y \\
\text { hospitalario) }\end{array}$ & $\begin{array}{l}\text { Farmacéuticos } \\
\text { profesionales }\end{array}$ & Experimental & DPC & ND & 51 & 14 horas & Presencial \\
\hline $\begin{array}{l}\text { Farrel, } \\
\text { et al. }{ }^{16}\end{array}$ & Inglaterra & $\begin{array}{c}\text { Ambos } \\
\text { (ambulatorio } \\
\text { y } \\
\text { hospitalario) }\end{array}$ & $\begin{array}{c}\text { Farmacéuticos } \\
\text { profesionales }\end{array}$ & Mixto & PEC & ND & 77 & 10 horas & Virtual \\
\hline $\begin{array}{l}\text { Farrel, } \\
\text { et al. } .^{17}\end{array}$ & Canadá & Ambulatorio & $\begin{array}{c}\text { Farmacéuticos } \\
\text { profesionales }\end{array}$ & Mixto & PEC & ND & 75 & 35 horas & $\begin{array}{l}\text { Virtual/ } \\
\text { Presencial }\end{array}$ \\
\hline $\begin{array}{l}\text { Buxton, } \\
\text { et al. }{ }^{18}\end{array}$ & $\begin{array}{l}\text { Estados } \\
\text { Unidos }\end{array}$ & Ambulatorio & $\begin{array}{c}\text { Personal } \\
\text { farmacéutico } \\
\text { (profesionales } \\
\text { y técnicos) }\end{array}$ & $\begin{array}{c}\text { Observacional } \\
\text { descriptivo }\end{array}$ & PEC & ND & 22 & 1,5 horas & Virtual \\
\hline $\begin{array}{l}\text { Hudmon, } \\
\text { et al. } .^{19}\end{array}$ & $\begin{array}{l}\text { Estados } \\
\text { Unidos }\end{array}$ & Ambulatorio & $\begin{array}{c}\text { Farmacéuticos } \\
\text { profesionales }\end{array}$ & $\begin{array}{c}\text { Cuasi- } \\
\text { experimental }\end{array}$ & PEC & ND & 819 & 2 horas & $\begin{array}{c}\text { Virtual/ } \\
\text { Presencial }\end{array}$ \\
\hline $\begin{array}{l}\text { Hughes, } \\
\text { et al. }{ }^{20}\end{array}$ & Canadá & $\begin{array}{c}\text { Ambos } \\
\text { (ambulatorio } \\
\text { y } \\
\text { hospitalario) }\end{array}$ & $\begin{array}{c}\text { Farmacéuticos } \\
\text { profesionales }\end{array}$ & Mixto & DPC & ND & 25 & ND & $\begin{array}{c}\text { Virtual/ } \\
\text { Presencial }\end{array}$ \\
\hline $\begin{array}{l}\text { Javadi, } \\
\text { et al. }{ }^{21}\end{array}$ & Irán & Ambulatorio & $\begin{array}{c}\text { Farmacéuticos } \\
\text { profesionales }\end{array}$ & Experimental & PEC & ND & 47 & 3 horas & Presencial \\
\hline $\begin{array}{l}\text { Sarayani, } \\
\text { et al. } .^{22}\end{array}$ & Irán & $\begin{array}{c}\text { Ambos } \\
\text { (ambulatorio } \\
\text { y } \\
\text { hospitalario) }\end{array}$ & $\begin{array}{c}\text { Farmacéuticos } \\
\text { profesionales }\end{array}$ & Experimental & PEC & ND & 139 & 4 horas & Virtual \\
\hline Dhital, et al. ${ }^{25}$ & Inglaterra & Ambulatorio & $\begin{array}{c}\text { Farmacéuticos } \\
\text { profesionales }\end{array}$ & Mixto & Entrenamiento & 27 & 29 & 8 horas & Presencial \\
\hline
\end{tabular}


Programas e intervenciones educativas ofrecidas al personal farmacéutico para mejorar la prestación de los servicios asistenciales farmacéuticos: una revisión estructurada

\begin{tabular}{|c|c|c|c|c|c|c|c|c|c|}
\hline Autor & País & Contexto & $\begin{array}{c}\text { Nivel de } \\
\text { formación }\end{array}$ & $\begin{array}{l}\text { Diseño del } \\
\text { estudio }\end{array}$ & $\begin{array}{c}\text { Tipo } \\
\text { intervención }\end{array}$ & $\begin{array}{c}\text { Cantidad de } \\
\text { farmacias } \\
\text { incluidas }\end{array}$ & $\begin{array}{l}\text { Cantidad de } \\
\text { farmacéuticos } \\
\text { intervenidos }\end{array}$ & $\begin{array}{c}\text { Tiempo de } \\
\text { duración } \\
\text { de la } \\
\text { intervención }\end{array}$ & $\begin{array}{c}\text { Métodos } \\
\text { de la } \\
\text { intervención }\end{array}$ \\
\hline $\begin{array}{l}\text { Meštrović, et } \\
\text { al. }^{26}\end{array}$ & Croacia & Ambulatorio & $\begin{array}{l}\text { Farmacéuticos } \\
\text { profesionales }\end{array}$ & $\begin{array}{l}\text { Observacional } \\
\text { analítico }\end{array}$ & Entrenamiento & 38 & 100 & 4 horas & Presencial \\
\hline $\begin{array}{l}\text { Terblanche, } \\
\text { et al. } .^{27}\end{array}$ & Sudáfrica & Hospitalario & $\begin{array}{l}\text { Farmacéuticos } \\
\text { profesionales }\end{array}$ & $\begin{array}{l}\text { Cuasi- } \\
\text { experimental }\end{array}$ & Entrenamiento & ND & 37 & ND & Presencial \\
\hline Minh, et al. ${ }^{28}$ & Vietnam & Ambulatorio & $\begin{array}{c}\text { Personal } \\
\text { farmacéutico } \\
\text { (profesionales y } \\
\text { técnicos) }\end{array}$ & $\begin{array}{l}\text { Cuasi- } \\
\text { experimental }\end{array}$ & Entrenamiento & 220 & 281 & $\begin{array}{l}24 \text { horas } \\
\text { (3 días) }\end{array}$ & Presencial \\
\hline $\begin{array}{l}\text { Barojek, et } \\
\text { al. }{ }^{29}\end{array}$ & Australia & Ambulatorio & $\begin{array}{c}\text { Personal } \\
\text { farmacéutico } \\
\text { (profesionales y } \\
\text { técnicos) }\end{array}$ & Mixto & Entrenamiento & ND & 17 & 8 horas & Presencial \\
\hline Pham, et al. ${ }^{30}$ & Vietnam & Ambulatorio & $\begin{array}{c}\text { Personal } \\
\text { farmacéutico } \\
\text { (profesionales y } \\
\text { técnicos) }\end{array}$ & $\begin{array}{l}\text { Cuasi- } \\
\text { experimental }\end{array}$ & Entrenamiento & 220 & 281 & 3,5 horas & Presencial \\
\hline $\begin{array}{l}\text { Ngwerume, } \\
\text { et al. }{ }^{31}\end{array}$ & Inglaterra & Ambulatorio & $\begin{array}{c}\text { Personal } \\
\text { farmacéutico } \\
\text { (profesionales y } \\
\text { técnicos) }\end{array}$ & $\begin{array}{c}\text { Cuasi- } \\
\text { experimental }\end{array}$ & Entrenamiento & 3 & 16 & 1,5 horas & Presencial \\
\hline $\begin{array}{l}\text { Chowdhury, } \\
\text { et al. }{ }^{32}\end{array}$ & Bangladesh & Ambulatorio & $\begin{array}{c}\text { Personal } \\
\text { farmacéutico } \\
\text { (profesionales y } \\
\text { técnicos) }\end{array}$ & $\begin{array}{c}\text { Cuasi- } \\
\text { experimental }\end{array}$ & Entrenamiento & 100 & 100 & 8 horas & Presencial \\
\hline Jha, et al. ${ }^{33}$ & Nepal & Ambulatorio & $\begin{array}{l}\text { Farmacéuticos } \\
\text { profesionales }\end{array}$ & $\begin{array}{l}\text { Cuasi- } \\
\text { experimental }\end{array}$ & Entrenamiento & 75 & 75 & 2 horas & Presencial \\
\hline $\begin{array}{l}\text { O'Neal, } \\
\text { et al. }{ }^{35}\end{array}$ & $\begin{array}{l}\text { Estados } \\
\text { Unidos }\end{array}$ & Ambulatorio & $\begin{array}{l}\text { Farmacéuticos } \\
\text { profesionales }\end{array}$ & Experimental & Entrenamiento & ND & 14 & 2 horas & Presencial \\
\hline Zillich, et al. ${ }^{36}$ & $\begin{array}{l}\text { Estados } \\
\text { Unidos }\end{array}$ & Ambulatorio & $\begin{array}{c}\text { Personal } \\
\text { farmacéutico } \\
\text { (profesionales y } \\
\text { técnicos) }\end{array}$ & $\begin{array}{c}\text { Observacional } \\
\text { descriptivo }\end{array}$ & Entrenamiento & 64 & 245 & ND & Presencial \\
\hline $\begin{array}{l}\text { Chartrand, } \\
\text { et al. }{ }^{37}\end{array}$ & Canadá & Ambulatorio & $\begin{array}{l}\text { Farmacéuticos } \\
\text { profesionales }\end{array}$ & $\begin{array}{c}\text { Cuasi- } \\
\text { experimental }\end{array}$ & Entrenamiento & 37 & 50 & ND & $\begin{array}{c}\text { Virtual/ } \\
\text { Presencial }\end{array}$ \\
\hline $\begin{array}{l}\text { Thomas, } \\
\text { et al. }^{38}\end{array}$ & $\begin{array}{l}\text { Estados } \\
\text { Unidos }\end{array}$ & Ambulatorio & $\begin{array}{l}\text { Farmacéuticos } \\
\text { profesionales }\end{array}$ & $\begin{array}{l}\text { Observacional } \\
\text { analítico }\end{array}$ & Entrenamiento & ND & 17 & ND & Presencial \\
\hline $\begin{array}{l}\text { Gheewala, } \\
\text { et al. }{ }^{39}\end{array}$ & Australia & Ambulatorio & $\begin{array}{l}\text { Farmacéuticos } \\
\text { profesionales }\end{array}$ & $\begin{array}{c}\text { Cuasi- } \\
\text { experimental }\end{array}$ & Entrenamiento & 27 & 50 & 1 hora & Virtual \\
\hline $\begin{array}{l}\text { O'Reilly, } \\
\text { et al. }\end{array}$ & Australia & Ambulatorio & $\begin{array}{l}\text { Farmacéuticos } \\
\text { profesionales }\end{array}$ & Mixto & Entrenamiento & 12 & 20 & 2 horas & Presencial \\
\hline $\begin{array}{l}\text { Palmer, } \\
\text { et al. }{ }^{42}\end{array}$ & $\begin{array}{l}\text { Estados } \\
\text { Unidos }\end{array}$ & $\begin{array}{c}\text { Ambos } \\
\text { (ambulatorio } \\
\text { y } \\
\text { hospitalario) }\end{array}$ & $\begin{array}{l}\text { Farmacéuticos } \\
\text { profesionales }\end{array}$ & $\begin{array}{c}\text { Observacional } \\
\text { descriptivo }\end{array}$ & Entrenamiento & ND & 1320 & 1,5 horas & $\begin{array}{l}\text { Virtual/ } \\
\text { Presencial }\end{array}$ \\
\hline $\begin{array}{l}\text { Guirguis, } \\
\text { et al. }\end{array}$ & Canadá & Ambulatorio & $\begin{array}{l}\text { Farmacéuticos } \\
\text { profesionales }\end{array}$ & $\begin{array}{l}\text { Cuasi- } \\
\text { experimental }\end{array}$ & Entrenamiento & ND & 42 & 1 hora & Presencial \\
\hline Laven, et al. ${ }^{44}$ & Alemania & Ambulatorio & $\begin{array}{l}\text { Farmacéuticos } \\
\text { profesionales }\end{array}$ & $\begin{array}{l}\text { Cuasi- } \\
\text { experimental }\end{array}$ & PEC & 18 & 26 & ND & $\begin{array}{c}\text { Virtual/ } \\
\text { Presencial }\end{array}$ \\
\hline $\begin{array}{l}\text { Bungard, } \\
\text { et al. }{ }^{45}\end{array}$ & Canadá & $\begin{array}{c}\text { Ambos } \\
\text { (ambulatorio } \\
y \\
\text { hospitalario) }\end{array}$ & $\begin{array}{l}\text { Farmacéuticos } \\
\text { profesionales }\end{array}$ & Mixto & PEC & ND & 71 & 14 horas & Presencial \\
\hline
\end{tabular}




\begin{tabular}{|c|c|c|c|c|c|c|c|c|c|}
\hline Autor & País & Contexto & $\begin{array}{c}\text { Nivel de } \\
\text { formación }\end{array}$ & $\begin{array}{l}\text { Diseño del } \\
\text { estudio }\end{array}$ & $\begin{array}{c}\text { Tipo } \\
\text { intervención }\end{array}$ & $\begin{array}{l}\text { Cantidad de } \\
\text { farmacias } \\
\text { incluidas }\end{array}$ & $\begin{array}{l}\text { Cantidad de } \\
\text { farmacéuticos } \\
\text { intervenidos }\end{array}$ & $\begin{array}{c}\text { Tiempo de } \\
\text { duración } \\
\text { de la } \\
\text { intervención }\end{array}$ & $\begin{array}{c}\text { Métodos } \\
\text { de la } \\
\text { intervención }\end{array}$ \\
\hline Fjortoft $^{46}$ & $\begin{array}{l}\text { Estados } \\
\text { Unidos }\end{array}$ & $\begin{array}{c}\text { Ambos } \\
\text { (ambulatorio } \\
\text { y } \\
\text { hospitalario) }\end{array}$ & $\begin{array}{l}\text { Farmacéuticos } \\
\text { profesionales }\end{array}$ & $\begin{array}{c}\text { Observacional } \\
\text { analítico }\end{array}$ & PEC & ND & 33 & $\begin{array}{l}12 \text { horas } \\
\text { ( } 1,5 \text { días })\end{array}$ & Presencial \\
\hline $\begin{array}{l}\text { Dimantouros, } \\
\text { et al. }{ }^{47}\end{array}$ & Canadá & $\begin{array}{c}\text { Ambos } \\
\text { (ambulatorio } \\
\text { y } \\
\text { hospitalario) }\end{array}$ & $\begin{array}{l}\text { Farmacéuticos } \\
\text { profesionales }\end{array}$ & $\begin{array}{l}\text { Cuasi- } \\
\text { experimental }\end{array}$ & PEC & ND & 21 & 15 horas & Presencial \\
\hline Kirk, et al. ${ }^{48}$ & $\begin{array}{l}\text { Estados } \\
\text { Unidos }\end{array}$ & Ambulatorio & $\begin{array}{l}\text { Farmacéuticos } \\
\text { profesionales }\end{array}$ & $\begin{array}{l}\text { Cuasi- } \\
\text { experimental }\end{array}$ & $\mathrm{PEC}$ & ND & 361 & 32 horas & Presencial \\
\hline $\begin{array}{l}\text { Moczygemba, } \\
\text { et al. }{ }^{49}\end{array}$ & $\begin{array}{l}\text { Estados } \\
\text { Unidos }\end{array}$ & $\begin{array}{c}\text { Ambos } \\
\text { (ambulatorio } \\
\text { y } \\
\text { hospitalario) }\end{array}$ & $\begin{array}{c}\text { Farmacéuticos } \\
\text { profesionales }\end{array}$ & $\begin{array}{c}\text { Observacional } \\
\text { analítico }\end{array}$ & PEC & ND & 23 & 120 horas & Virtual \\
\hline Martin, et al. ${ }^{50}$ & $\begin{array}{l}\text { Estados } \\
\text { Unidos }\end{array}$ & Ambulatorio & $\begin{array}{l}\text { Farmacéuticos } \\
\text { profesionales }\end{array}$ & $\begin{array}{l}\text { Cuasi- } \\
\text { experimental }\end{array}$ & PEC & ND & 25 & 10 horas & $\begin{array}{c}\text { Virtual/ } \\
\text { Presencial }\end{array}$ \\
\hline $\begin{array}{l}\text { Stafford, } \\
\text { et al. }\end{array}$ & Australia & Ambulatorio & $\begin{array}{l}\text { Farmacéuticos } \\
\text { profesionales }\end{array}$ & $\begin{array}{c}\text { Observacional } \\
\text { descriptivo }\end{array}$ & PEC & ND & 62 & 2 horas & Presencial \\
\hline Legris $^{52}$ & Canadá & Ambulatorio & $\begin{array}{l}\text { Farmacéuticos } \\
\text { profesionales }\end{array}$ & Experimental & PEC & 33 & 52 & 1 hora & Virtual \\
\hline Lee, et al. ${ }^{54}$ & Malasia & Ambulatorio & $\begin{array}{l}\text { Farmacéuticos } \\
\text { profesionales }\end{array}$ & $\begin{array}{l}\text { Cuasi- } \\
\text { experimental }\end{array}$ & PEC & ND & 40 & 5 horas & Presencial \\
\hline $\begin{array}{l}\text { Svetlana, } \\
\text { et al. } .^{55}\end{array}$ & Serbia & Ambulatorio & $\begin{array}{l}\text { Farmacéuticos } \\
\text { profesionales }\end{array}$ & $\begin{array}{l}\text { Cuasi- } \\
\text { experimental }\end{array}$ & Entrenamiento & 21 & 32 & ND & Presencial \\
\hline $\begin{array}{l}\text { Lalonde, } \\
2017^{56}\end{array}$ & Canadá & $\begin{array}{c}\text { Ambos } \\
\text { (ambulatorio } \\
\text { y } \\
\text { hospitalario) }\end{array}$ & $\begin{array}{l}\text { Farmacéuticos } \\
\text { profesionales }\end{array}$ & Experimental & PEC & 207 & 345 & 1,5 horas & Presencial \\
\hline Adepu, et al. ${ }^{63}$ & India & Ambulatorio & $\begin{array}{c}\text { Farmacéuticos } \\
\text { profesionales }\end{array}$ & $\begin{array}{c}\text { Cuasi- } \\
\text { experimental }\end{array}$ & DPC & ND & 48 & ND & Presencial \\
\hline $\begin{array}{l}\text { Augustine, } \\
\text { et al. }{ }^{64}\end{array}$ & $\begin{array}{l}\text { Estados } \\
\text { Unidos }\end{array}$ & Hospitalario & $\begin{array}{l}\text { Farmacéuticos } \\
\text { profesionales }\end{array}$ & $\begin{array}{c}\text { Observacional } \\
\text { descriptivo }\end{array}$ & PEC & ND & 63 & 4 horas & Presencial \\
\hline Batra, et al. ${ }^{65}$ & $\begin{array}{l}\text { Estados } \\
\text { Unidos }\end{array}$ & Ambulatorio & $\begin{array}{c}\text { Personal } \\
\text { farmacéutico } \\
\text { (profesionales y } \\
\text { técnicos) }\end{array}$ & $\begin{array}{l}\text { Observacional } \\
\text { descriptivo }\end{array}$ & PEC & 55 & 448 & 1 hora & Virtual \\
\hline $\begin{array}{l}\text { Buxton, } \\
\text { et al. }{ }^{66}\end{array}$ & $\begin{array}{l}\text { Estados } \\
\text { Unidos }\end{array}$ & Ambulatorio & $\begin{array}{l}\text { Farmacéuticos } \\
\text { profesionales }\end{array}$ & $\begin{array}{l}\text { Observacional } \\
\text { descriptivo }\end{array}$ & PEC & ND & 97 & 2 horas & Virtual \\
\hline $\begin{array}{l}\text { Buxton, } \\
\text { et al. }{ }^{67}\end{array}$ & $\begin{array}{l}\text { Estados } \\
\text { Unidos }\end{array}$ & Ambulatorio & $\begin{array}{l}\text { Farmacéuticos } \\
\text { profesionales }\end{array}$ & $\begin{array}{c}\text { Observacional } \\
\text { descriptivo }\end{array}$ & PEC & ND & 82 & 8 horas & Virtual \\
\hline $\begin{array}{l}\text { Charpentier, } \\
\text { et al. }{ }^{68}\end{array}$ & $\begin{array}{l}\text { Estados } \\
\text { Unidos }\end{array}$ & $\begin{array}{c}\text { Ambos } \\
\text { (ambulatorio } \\
y \\
\text { hospitalario) }\end{array}$ & $\begin{array}{c}\text { Personal } \\
\text { farmacéutico } \\
\text { (profesionales y } \\
\text { técnicos) }\end{array}$ & $\begin{array}{c}\text { Cuasi- } \\
\text { experimental }\end{array}$ & PEC & ND & 410 & 1,5 horas & Presencial \\
\hline $\begin{array}{l}\text { Chiang, } \\
\text { et al. }{ }^{69}\end{array}$ & Taiwán & $\begin{array}{c}\text { Ambos } \\
\text { (ambulatorio } \\
\text { y } \\
\text { hospitalario) }\end{array}$ & $\begin{array}{c}\text { Farmacéuticos } \\
\text { profesionales }\end{array}$ & $\begin{array}{c}\text { Cuasi- } \\
\text { experimental }\end{array}$ & PEC & ND & 125 & 20 horas & Presencial \\
\hline $\begin{array}{l}\text { Condren, } \\
\text { et al. }{ }^{70}\end{array}$ & $\begin{array}{l}\text { Estados } \\
\text { Unidos }\end{array}$ & Ambulatorio & $\begin{array}{l}\text { Farmacéuticos } \\
\text { profesionales }\end{array}$ & $\begin{array}{c}\text { Observacional } \\
\text { descriptivo }\end{array}$ & PEC & 46 & 100 & ND & Presencial \\
\hline $\begin{array}{l}\text { Coombes, } \\
\text { et al. }{ }^{71}\end{array}$ & Australia & Hospitalario & $\begin{array}{l}\text { Farmacéuticos } \\
\text { profesionales }\end{array}$ & $\begin{array}{l}\text { Cuasi- } \\
\text { experimental }\end{array}$ & Entrenamiento & ND & 66 & ND & Presencial \\
\hline $\begin{array}{l}\text { Donyai, } \\
\text { et al. }{ }^{72}\end{array}$ & Inglaterra & $\begin{array}{c}\text { Ambos } \\
\text { (ambulatorio } \\
y \\
\text { hospitalario) }\end{array}$ & $\begin{array}{c}\text { Personal } \\
\text { farmacéutico } \\
\text { (profesionales y } \\
\text { técnicos) }\end{array}$ & Experimental & DPC & ND & 31 & ND & $\begin{array}{c}\text { Virtual/ } \\
\text { Presencial }\end{array}$ \\
\hline
\end{tabular}


Programas e intervenciones educativas ofrecidas al personal farmacéutico para mejorar la prestación de los servicios asistenciales farmacéuticos: una revisión estructurada

\begin{tabular}{|c|c|c|c|c|c|c|c|c|c|}
\hline Autor & País & Contexto & $\begin{array}{c}\text { Nivel de } \\
\text { formación }\end{array}$ & $\begin{array}{l}\text { Diseño del } \\
\text { estudio }\end{array}$ & $\begin{array}{c}\text { Tipo } \\
\text { intervención }\end{array}$ & $\begin{array}{c}\text { Cantidad de } \\
\text { farmacias } \\
\text { incluidas }\end{array}$ & $\begin{array}{l}\text { Cantidad de } \\
\text { farmacéuticos } \\
\text { intervenidos }\end{array}$ & $\begin{array}{c}\text { Tiempo de } \\
\text { duración } \\
\text { de la } \\
\text { intervención }\end{array}$ & $\begin{array}{c}\text { Métodos } \\
\text { de la } \\
\text { intervención }\end{array}$ \\
\hline Dopp, et al. ${ }^{73}$ & $\begin{array}{l}\text { Estados } \\
\text { Unidos }\end{array}$ & $\begin{array}{c}\text { Ambos } \\
\text { (ambulatorio } \\
\text { y } \\
\text { hospitalario) }\end{array}$ & $\begin{array}{c}\text { Farmacéuticos } \\
\text { profesionales }\end{array}$ & $\begin{array}{l}\text { Observacional } \\
\text { analítico }\end{array}$ & $\mathrm{DPC}$ & ND & 127 & 19 horas & Presencial \\
\hline $\begin{array}{l}\text { Dualde, } \\
\text { et al. }{ }^{74}\end{array}$ & España & Ambulatorio & $\begin{array}{l}\text { Farmacéuticos } \\
\text { profesionales }\end{array}$ & $\begin{array}{c}\text { Observacional } \\
\text { descriptivo }\end{array}$ & Entrenamiento & ND & 225 & 16 horas & Presencial \\
\hline $\begin{array}{l}\text { Dualde, } \\
\text { et al. }{ }^{75}\end{array}$ & España & Ambulatorio & $\begin{array}{c}\text { Farmacéuticos } \\
\text { profesionales }\end{array}$ & $\begin{array}{c}\text { Observacional } \\
\text { descriptivo }\end{array}$ & PEC & ND & 10981 & ND & Presencial \\
\hline Elaro, et al. ${ }^{76}$ & Australia & Ambulatorio & $\begin{array}{l}\text { Farmacéuticos } \\
\text { profesionales }\end{array}$ & Mixto & PEC & 37 & 44 & 6 horas & Presencial \\
\hline Feletto, et al. ${ }^{77}$ & Australia & Ambulatorio & $\begin{array}{c}\text { Personal } \\
\text { farmacéutico } \\
\text { (profesionales y } \\
\text { técnicos) }\end{array}$ & Mixto & PEC & 37 & 45 & $\begin{array}{l}16 \text { horas } \\
\text { ( } 2 \text { días) }\end{array}$ & Presencial \\
\hline $\begin{array}{l}\text { Fitzgerald, et } \\
\text { al. }{ }^{78}\end{array}$ & Escocia & Ambulatorio & $\begin{array}{l}\text { Farmacéuticos } \\
\text { profesionales }\end{array}$ & $\begin{array}{l}\text { Cuasi- } \\
\text { experimental }\end{array}$ & Entrenamiento & 8 & 8 & $\begin{array}{l}16 \text { horas } \\
\text { ( } 2 \text { días) }\end{array}$ & Presencial \\
\hline $\begin{array}{l}\text { Formea, et } \\
\text { al. } .^{79}\end{array}$ & $\begin{array}{l}\text { Estados } \\
\text { Unidos }\end{array}$ & $\begin{array}{c}\text { Ambos } \\
\text { (ambulatorio } \\
\text { y } \\
\text { hospitalario) }\end{array}$ & $\begin{array}{l}\text { Farmacéuticos } \\
\text { profesionales }\end{array}$ & $\begin{array}{l}\text { Cuasi- } \\
\text { experimental }\end{array}$ & PEC & 6 & 186 & 1 hora & $\begin{array}{c}\text { Virtual/ } \\
\text { Presencial }\end{array}$ \\
\hline Garcia, et al. ${ }^{80}$ & Perú & Ambulatorio & $\begin{array}{c}\text { Personal } \\
\text { farmacéutico } \\
\text { (profesionales y } \\
\text { técnicos) }\end{array}$ & Experimental & PEC & 696 & 2074 & 60 horas & Presencial \\
\hline $\begin{array}{l}\text { Grzeskowiak, } \\
\text { et al. }{ }^{81}\end{array}$ & Australia & Hospitalario & $\begin{array}{l}\text { Farmacéuticos } \\
\text { profesionales }\end{array}$ & Experimental & PEC & 2 & 44 & $\begin{array}{l}\text { Menos de } \\
1 \text { hora }\end{array}$ & Presencial \\
\hline Kjome, et al. ${ }^{83}$ & Noruega & Ambulatorio & $\begin{array}{l}\text { Farmacéuticos } \\
\text { profesionales }\end{array}$ & $\begin{array}{l}\text { Observacional } \\
\text { analítico }\end{array}$ & Entrenamiento & 16 & 16 & ND & Presencial \\
\hline $\begin{array}{l}\text { Kouladjian, } \\
\text { et al. }{ }^{84}\end{array}$ & Australia & Ambulatorio & $\begin{array}{l}\text { Farmacéuticos } \\
\text { profesionales }\end{array}$ & $\begin{array}{c}\text { Observacional } \\
\text { descriptivo }\end{array}$ & $\mathrm{DPC}$ & ND & 2576 & ND & Presencial \\
\hline $\begin{array}{l}\text { Kristina, } \\
\text { et al. }{ }^{85}\end{array}$ & Indonesia & Ambulatorio & $\begin{array}{l}\text { Farmacéuticos } \\
\text { profesionales }\end{array}$ & $\begin{array}{l}\text { Cuasi- } \\
\text { experimental }\end{array}$ & Entrenamiento & ND & 133 & 6 horas & Presencial \\
\hline $\begin{array}{l}\text { Kuo, et al. } \\
2013^{86}\end{array}$ & $\begin{array}{l}\text { Estados } \\
\text { Unidos }\end{array}$ & $\begin{array}{c}\text { Ambos } \\
\text { (ambulatorio } \\
\text { y } \\
\text { hospitalario) }\end{array}$ & $\begin{array}{l}\text { Farmacéuticos } \\
\text { profesionales }\end{array}$ & $\begin{array}{l}\text { Cuasi- } \\
\text { experimental }\end{array}$ & PEC & ND & 673 & 2 horas & Presencial \\
\hline $\begin{array}{l}\text { Laaksonen, } \\
\text { et al. }{ }^{87}\end{array}$ & Inglaterra & Ambulatorio & $\begin{array}{c}\text { Farmacéuticos } \\
\text { profesionales }\end{array}$ & $\begin{array}{c}\text { Observacional } \\
\text { descriptivo }\end{array}$ & Entrenamiento & ND & 43 & $\begin{array}{l}24 \text { horas } \\
\text { ( } 3 \text { días) }\end{array}$ & Presencial \\
\hline $\begin{array}{l}\text { Laaksonen, } \\
\text { et al. }{ }^{88}\end{array}$ & Inglaterra & Ambulatorio & $\begin{array}{l}\text { Farmacéuticos } \\
\text { profesionales }\end{array}$ & $\begin{array}{c}\text { Observacional } \\
\text { descriptivo }\end{array}$ & Entrenamiento & 43 & 43 & 60 horas & Virtual \\
\hline Lalonde. ${ }^{89}$ & Canadá & $\begin{array}{c}\text { Ambos } \\
\text { (ambulatorio } \\
\text { y } \\
\text { hospitalario) }\end{array}$ & $\begin{array}{l}\text { Farmacéuticos } \\
\text { profesionales }\end{array}$ & Experimental & PEC & 42 & 50 & 3 horas & Presencial \\
\hline $\begin{array}{l}\text { Liekens, } \\
\text { et al. }{ }^{90}\end{array}$ & Bélgica & Ambulatorio & $\begin{array}{l}\text { Farmacéuticos } \\
\text { profesionales }\end{array}$ & Experimental & Entrenamiento & ND & 90 & 1,5 horas & Presencial \\
\hline $\begin{array}{l}\text { Liekens, } \\
\text { et al. }{ }^{91}\end{array}$ & Bélgica & Ambulatorio & $\begin{array}{l}\text { Farmacéuticos } \\
\text { profesionales }\end{array}$ & Experimental & Entrenamiento & ND & 21 & $\begin{array}{l}8 \text { horas } \\
\text { (1 día) }\end{array}$ & Presencial \\
\hline Martin, et al. ${ }^{92}$ & $\begin{array}{l}\text { Estados } \\
\text { Unidos }\end{array}$ & Ambulatorio & $\begin{array}{c}\text { Personal } \\
\text { farmacéutico } \\
\text { (profesionales y } \\
\text { técnicos) }\end{array}$ & $\begin{array}{c}\text { Cuasi- } \\
\text { experimental }\end{array}$ & Entrenamiento & ND & 25 & 8 horas & Presencial \\
\hline
\end{tabular}




\begin{tabular}{|c|c|c|c|c|c|c|c|c|c|}
\hline Autor & País & Contexto & $\begin{array}{c}\text { Nivel de } \\
\text { formación }\end{array}$ & $\begin{array}{l}\text { Diseño del } \\
\text { estudio }\end{array}$ & $\begin{array}{c}\text { Tipo } \\
\text { intervención }\end{array}$ & $\begin{array}{l}\text { Cantidad de } \\
\text { farmacias } \\
\text { incluidas }\end{array}$ & $\begin{array}{l}\text { Cantidad de } \\
\text { farmacéuticos } \\
\text { intervenidos }\end{array}$ & $\begin{array}{c}\text { Tiempo de } \\
\text { duración } \\
\text { de la } \\
\text { intervención }\end{array}$ & $\begin{array}{c}\text { Métodos } \\
\text { de la } \\
\text { intervención }\end{array}$ \\
\hline $\begin{array}{l}\text { McConnell, } \\
\text { et al. }{ }^{93}\end{array}$ & $\begin{array}{l}\text { Estados } \\
\text { Unidos }\end{array}$ & $\begin{array}{c}\text { Ambos } \\
\text { (ambulatorio } \\
y \\
\text { hospitalario) }\end{array}$ & $\begin{array}{c}\text { Farmacéuticos } \\
\text { profesionales }\end{array}$ & Experimental & $\mathrm{DPC}$ & ND & 51 & 14 horas & Presencial \\
\hline $\begin{array}{l}\text { McConnell, } \\
\text { et al. }{ }^{94}\end{array}$ & $\begin{array}{l}\text { Estados } \\
\text { Unidos }\end{array}$ & $\begin{array}{c}\text { Ambos } \\
\text { (ambulatorio } \\
y \\
\text { hospitalario) }\end{array}$ & $\begin{array}{c}\text { Farmacéuticos } \\
\text { profesionales }\end{array}$ & $\begin{array}{c}\text { Observacional } \\
\text { descriptivo }\end{array}$ & $\mathrm{DPC}$ & ND & 51 & 14 horas & Presencial \\
\hline Melo, et al. ${ }^{95}$ & Brasil & Hospitalario & $\begin{array}{c}\text { Técnicos } \\
\text { (solamente) }\end{array}$ & $\begin{array}{c}\text { Observacional } \\
\text { descriptivo }\end{array}$ & Entrenamiento & ND & 4 & 8 horas & Presencial \\
\hline $\begin{array}{l}\text { O'breli-Neto, } \\
\text { et al. }{ }^{96}\end{array}$ & Brasil & Ambulatorio & $\begin{array}{c}\text { Farmacéuticos } \\
\text { profesionales }\end{array}$ & Experimental & PEC & 185 & 61 & ND & Virtual \\
\hline $\begin{array}{l}\text { O'Neal, } \\
\text { et al. }{ }^{97}\end{array}$ & $\begin{array}{l}\text { Estados } \\
\text { Unidos }\end{array}$ & Ambulatorio & $\begin{array}{c}\text { Personal } \\
\text { farmacéutico } \\
\text { (profesionales y } \\
\text { técnicos) }\end{array}$ & $\begin{array}{l}\text { Observacional } \\
\text { descriptivo }\end{array}$ & PEC & 8 & 31 & 2 horas & Presencial \\
\hline $\begin{array}{l}\text { Rouleau, } \\
\text { et al. }{ }^{98}\end{array}$ & Canadá & Ambulatorio & $\begin{array}{c}\text { Personal } \\
\text { farmacéutico } \\
\text { (profesionales y } \\
\text { técnicos) }\end{array}$ & Experimental & PEC & ND & 52 & 2 horas & Presencial \\
\hline Sadler, et al. ${ }^{99}$ & $\begin{array}{l}\text { Estados } \\
\text { Unidos }\end{array}$ & Ambulatorio & $\begin{array}{c}\text { Farmacéuticos } \\
\text { profesionales }\end{array}$ & $\begin{array}{c}\text { Observacional } \\
\text { descriptivo }\end{array}$ & Entrenamiento & ND & 6 & 22 horas & Presencial \\
\hline Saw, et al. ${ }^{100}$ & Malasia & Ambulatorio & $\begin{array}{c}\text { Farmacéuticos } \\
\text { profesionales }\end{array}$ & $\begin{array}{c}\text { Cuasi- } \\
\text { experimental }\end{array}$ & Entrenamiento & ND & 37 & ND & Presencial \\
\hline $\begin{array}{l}\text { Saylor, et } \\
\text { al. }{ }^{101}\end{array}$ & $\begin{array}{l}\text { Estados } \\
\text { Unidos }\end{array}$ & Hospitalario & $\begin{array}{c}\text { Farmacéuticos } \\
\text { profesionales }\end{array}$ & $\begin{array}{l}\text { Observacional } \\
\text { analítico }\end{array}$ & Entrenamiento & ND & 59 & ND & Presencial \\
\hline Sidhu, et al. ${ }^{103}$ & Canadá & Hospitalario & $\begin{array}{l}\text { Farmacéuticos } \\
\text { profesionales }\end{array}$ & $\begin{array}{c}\text { Cuasi- } \\
\text { experimental }\end{array}$ & DPC & ND & 58 & 1,5 horas & Presencial \\
\hline Smith, et al. ${ }^{104}$ & Australia & Ambulatorio & $\begin{array}{c}\text { Farmacéuticos } \\
\text { profesionales }\end{array}$ & $\begin{array}{c}\text { Cuasi- } \\
\text { experimental }\end{array}$ & DPC & ND & 292 & ND & Presencial \\
\hline Sweet, et al. ${ }^{105}$ & $\begin{array}{l}\text { Estados } \\
\text { Unidos }\end{array}$ & Ambulatorio & $\begin{array}{c}\text { Farmacéuticos } \\
\text { profesionales }\end{array}$ & $\begin{array}{l}\text { Cuasi- } \\
\text { experimental }\end{array}$ & PEC & ND & 29 & 1 hora & Virtual \\
\hline $\begin{array}{l}\text { Temple, } \\
\text { et al. }{ }^{106}\end{array}$ & $\begin{array}{l}\text { Estados } \\
\text { Unidos }\end{array}$ & Hospitalario & $\begin{array}{c}\text { Farmacéuticos } \\
\text { profesionales }\end{array}$ & $\begin{array}{l}\text { Cuasi- } \\
\text { experimental }\end{array}$ & Entrenamiento & ND & 7 & 57 horas & Presencial \\
\hline $\begin{array}{l}\text { Tofade, } \\
\text { et al. }{ }^{107}\end{array}$ & $\begin{array}{l}\text { Estados } \\
\text { Unidos }\end{array}$ & Ambulatorio & $\begin{array}{c}\text { Farmacéuticos } \\
\text { profesionales }\end{array}$ & $\begin{array}{c}\text { Cuasi- } \\
\text { experimental }\end{array}$ & DPC & ND & 120 & 5 horas & Presencial \\
\hline $\begin{array}{l}\text { Tofade, } \\
\text { et al. }{ }^{108}\end{array}$ & $\begin{array}{l}\text { Estados } \\
\text { Unidos }\end{array}$ & $\begin{array}{c}\text { Ambos } \\
\text { (ambulatorio } \\
\text { y } \\
\text { hospitalario) }\end{array}$ & $\begin{array}{c}\text { Farmacéuticos } \\
\text { profesionales }\end{array}$ & $\begin{array}{c}\text { Cuasi- } \\
\text { experimental }\end{array}$ & $\mathrm{DPC}$ & ND & 21 & 5 horas & Presencial \\
\hline $\begin{array}{l}\text { Trewet, } \\
\text { et al. }{ }^{109}\end{array}$ & $\begin{array}{l}\text { Estados } \\
\text { Unidos }\end{array}$ & Ambulatorio & $\begin{array}{l}\text { Farmacéuticos } \\
\text { profesionales }\end{array}$ & $\begin{array}{l}\text { Observacional } \\
\text { analítico }\end{array}$ & $\mathrm{DPC}$ & ND & 47 & ND & Presencial \\
\hline Wahl, et al. ${ }^{110}$ & $\begin{array}{l}\text { Estados } \\
\text { Unidos }\end{array}$ & Ambulatorio & $\begin{array}{c}\text { Farmacéuticos } \\
\text { profesionales }\end{array}$ & $\begin{array}{c}\text { Observacional } \\
\text { descriptivo }\end{array}$ & Entrenamiento & ND & 37 & ND & Presencial \\
\hline $\begin{array}{l}\text { Walters, } \\
\text { et al. }{ }^{111}\end{array}$ & $\begin{array}{l}\text { Nueva } \\
\text { Zelanda }\end{array}$ & Ambulatorio & $\begin{array}{c}\text { Farmacéuticos } \\
\text { profesionales }\end{array}$ & $\begin{array}{l}\text { Cuasi- } \\
\text { experimental }\end{array}$ & Entrenamiento & ND & 190 & ND & Virtual \\
\hline $\begin{array}{l}\text { Wheeler, } \\
\text { et al. }{ }^{112}\end{array}$ & Australia & Ambulatorio & $\begin{array}{c}\text { Personal } \\
\text { farmacéutico } \\
\text { (profesionales y } \\
\text { técnicos) }\end{array}$ & $\begin{array}{c}\text { Cuasi- } \\
\text { experimental }\end{array}$ & PEC & ND & 504 & 4 horas & $\begin{array}{c}\text { Virtual/ } \\
\text { Presencial }\end{array}$ \\
\hline $\begin{array}{l}\text { Wilbur, } \\
\text { et al. }{ }^{113}\end{array}$ & Qatar & Ambulatorio & $\begin{array}{l}\text { Farmacéuticos } \\
\text { profesionales }\end{array}$ & Cualitativo & PEC & ND & 41 & 1 hora & Presencial \\
\hline
\end{tabular}




\section{Características de los diseños y metodologías de los estudios}

De los 90 estudios, $60(67 \%)$ se realizaron a nivel ambulatorio, $9(10 \%)$ a nivel hospitalario, y $21(23 \%)$ en ambos contextos. Adicionalmente, 72 (80\%) describieron, en su metodología, la estructura de la intervención, así como los temas, contenidos y/o los procesos abordados. En los estudios con dos o más mediciones, el tiempo de seguimiento varió de 1 a 44 meses, con una mediana de 6 meses (RIC 9,8). Sin embargo, 8 estudios no describieron el tiempo entre las mediciones y tres estudios describieron que las mediciones se realizaron inmediatamente después de la intervención.

\section{Características de las farmacias y del personal farmacéuticos incluidos en los estudios}

La mediana de la cantidad de farmacias que fueron invitadas a participar en los estudios fue de 80 (RIC 216) y la proporción de participación solo se encontró descrita en 23 estudios $(26 \%)$. En tres de ellos, el porcentaje de participación fue de $100 \%$ (2, 6 y 21 farmacias); para las demás, la proporción de participación varió entre $0,6 \%$ y $90 \%$. El estudio con mayor cantidad de farmacias invitadas a participar reportó 3075 farmacias, de las cuales solo participaron 18, siendo el estudio con menor porcentaje de participación $(0,6 \%)$, debido principalmente al costo y a la inversión de tiempo, los que fueron más altos de lo habitual para un programa de educación continua.

De los 90 estudios, en 42 (47\%) reportaron un rango entre 27 a 10891 como personal farmacéutico invitado a participar; mientras que $33(37 \%)$ reportaron un rango entre 16 a 1245 personas. En este sentido, en 20 (22\%) de estos estudios, en los cuales se describieron ambos valores, se calculó la proporción de participación del personal farmacéutico, el cual varió entre $20 \%$ y $100 \%$, con una mediana de $62 \%$ (RIC 58). En 26 (29\%) estudios, los autores describieron el total de personal farmacéutico que terminó el estudio, sin importar si recibieron o no la intervención. Acorde con este dato y con la cantidad de personal farmacéutico que inició el estudio (en 23 estudios que lo reportaron y que tenían 2 o más mediciones), las perdidas en el seguimiento variaron desde $0 \%$ (en 8 estudios), hasta $75 \%$, con una mediana de $9 \%$ (RIC 31). De los 23 estudios en los que se calculó este valor, 16 de ellos eran estudios experimentales y la mediana en las perdidas en el seguimiento fue de $9 \%$ (RIC 31). Adicional al personal farmacéutico, 5 estudios incluyeron otros profesionales de la salud, entre ellos, médicos, residentes y enfermeras.
Por otro lado, en 18 estudios se evaluó la efectividad de la intervención también en pacientes, hallándose un promedio de 325 pacientes, en un rango entre 59 a 1006 pacientes evaluados. Las características adicionales sobre metodologías, farmacias y personal farmacéutico se describen en la Tabla 2.

\section{Objetivos evaluados en los estudios}

Los objetivos principales de los 90 estudios se centraron en el desarrollo (descripción) y la evaluación del impacto, la efectividad (efecto) o la comparación de una intervención educativa enfocada en un entrenamiento específico (41\%), un programa de EC $(43 \%)$ o un programa de DPC (16\%) dirigido al personal farmacéutico.

En 14 estudios (16\%) realizaron la evaluación de las necesidades de aprendizaje, educación y capacitación del personal farmacéutico y la utilización de estos hallazgos para el desarrollo de las intervenciones o los programas educativos; sin embargo, en el texto de los artículos no se identificó dicha información. Solo un grupo de investigadores informó los resultados de la evaluación de la efectividad del programa de EC, posterior a la realización y publicación del estudio piloto del mismo, teniendo en cuenta el rango de tiempo que se tomó para la revisión ${ }^{15,16}$. Además, en cuatro estudios los investigadores compararon dos o más metodologías diferentes o formas de entrega del mismo programa de $\mathrm{EC}^{17-20}, \mathrm{y}$ un estudio evaluó la eficacia comparativa de tres diferentes programas de EC como intervención a un mismo problema salud ${ }^{21}$.

\section{Problemas de salud y enfermedades intervenidos en los programas educativos}

Los estudios se focalizaron en el entrenamiento y desarrollo de herramientas, estrategias, materiales, contenidos educativos y de aprendizaje para suplir las necesidades en el personal farmacéutico de diferentes problemas de salud, de servicios asistenciales farmacéuticos y de los procesos técnicos y administrativos de las farmacias para su correcta gestión. Un 70\% (63) de los estudios fueron dirigidos a uno o varios problemas de salud específicos: 47 estudios en un solo problema de salud, y 16 en dos o más problemas de salud diferentes. De forma similar, algunas intervenciones tenían como parte de sus contenidos un grupo farmacológico de medicamentos específico de uso terapéutico para uno o varios problemas de salud. En la Tabla 3 se describen los problemas de salud y enfermedades intervenidos. 
Tabla 2. Características de los diseños y metodologías de los estudios.

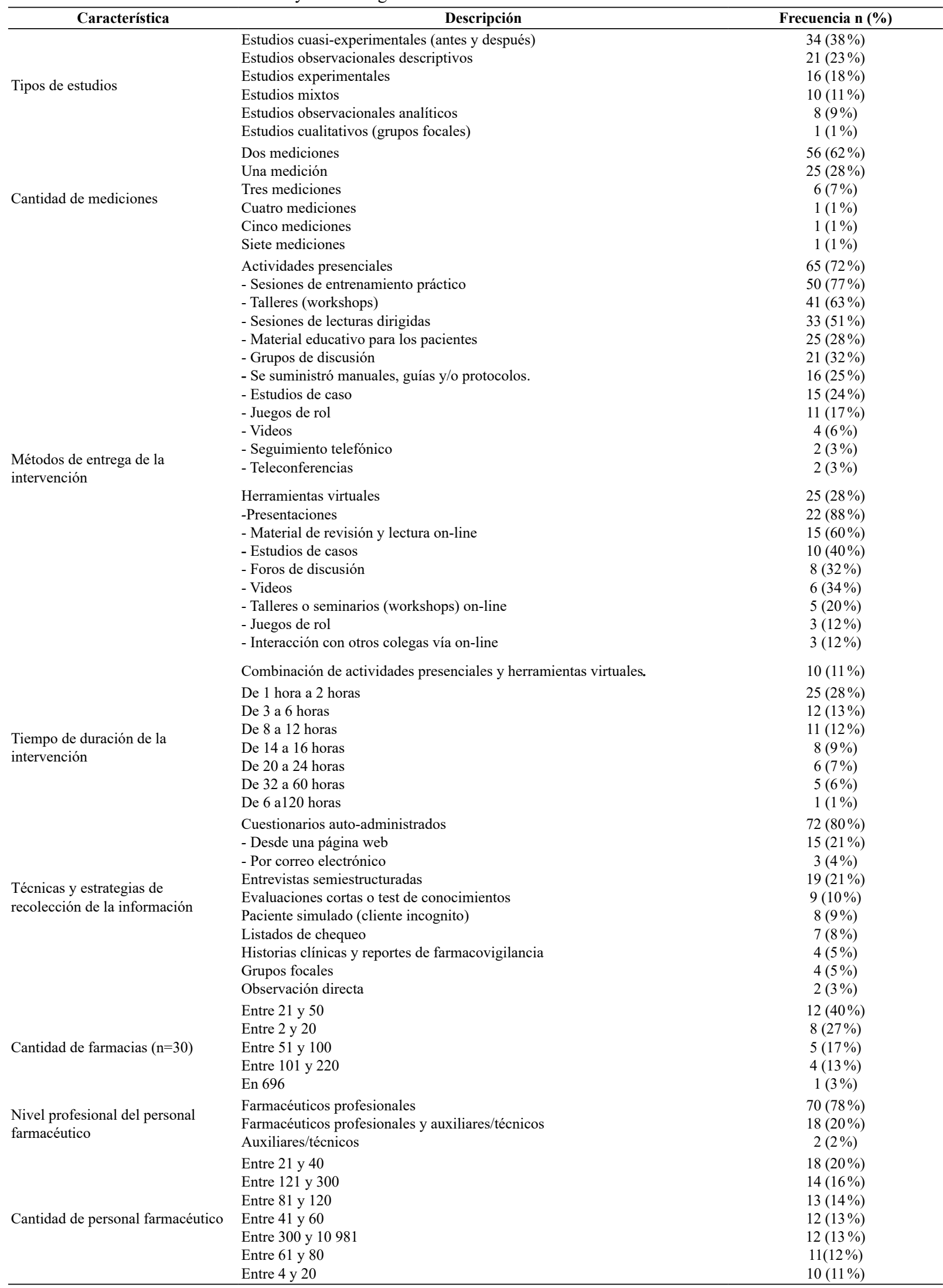


Tabla 3. Problemas de salud y enfermedades intervenidos en los programas educativos.

\begin{tabular}{|c|c|}
\hline Problema de salud & Frecuencia n $(\%)$ \\
\hline Salud mental & $16(18 \%)$ \\
\hline - Depresión & $5(6 \%)$ \\
\hline - Control y prevención del tabaquismo & $5(6 \%)$ \\
\hline - Problemas con el alcohol & $2(3 \%)$ \\
\hline - Ansiedad & $1(1 \%)$ \\
\hline - Prevención de sustancias de abuso & $1(1 \%)$ \\
\hline - Sustitución de opioides & $1(1 \%)$ \\
\hline - Abuso de medicamentos & $1(1 \%)$ \\
\hline Salud sexual y reproductiva & $6(7 \%)$ \\
\hline Problemas de coagulación sanguínea & $6(7 \%)$ \\
\hline Asma & $5(6 \%)$ \\
\hline Enfermedad renal crónica & $4(5 \%)$ \\
\hline Cáncer & $4(5 \%)$ \\
\hline Prevención de embarazos & $3(4 \%)$ \\
\hline Diabetes y medición de la glucosa & $3(4 \%)$ \\
\hline Enfermedades cardiovasculares & $3(4 \%)$ \\
\hline Farmacogenómica & $3(4 \%)$ \\
\hline Enfermedades gastrointestinales & $3(4 \%)$ \\
\hline Enfermedades infecciosas & $3(4 \%)$ \\
\hline Manejo de dolencias menores (uso de medicamentos de venta libre) & $3(4 \%)$ \\
\hline Embarazos de alto riesgo materno, neonatos y pediatría & $2(3 \%)$ \\
\hline Diarrea & $2(3 \%)$ \\
\hline Enfermedades endocrinas & $2(3 \%)$ \\
\hline Control del dolor & $2(3 \%)$ \\
\hline Disfunción sexual & $1(1 \%)$ \\
\hline Enfermedades de transmisión sexual & $1(1 \%)$ \\
\hline Enfermedades esqueléticas & $1(1 \%)$ \\
\hline
\end{tabular}

\section{Servicios asistenciales farmacéuticos intervenidos en los programas educativos}

De los 90 estudios, 10 (11\%) abordaron varios de los servicios asistenciales farmacéuticos, como dispensación, seguimiento farmacoterapéutico y monitorización, educación/consejería, interacciones medicamentosas, reacciones adversas, cálculos de dosis, entre otros. En la Tabla 4 se describen los procesos intervenidos en los programas educativos.

Tabla 4. Servicios asistenciales farmacéuticos intervenidos en los programas educativos.

\begin{tabular}{|c|c|c|}
\hline Característica & Descripción & Frecuencia n (\%) \\
\hline \multirow{5}{*}{$\begin{array}{l}\text { Servicios asistenciales } \\
\text { farmacéuticos }\end{array}$} & $\begin{array}{l}\text { Seguimiento y monitorización (seguimiento farmacoterapéutico), } \\
\text { cálculo de dosis. }\end{array}$ & $57(63 \%)$ \\
\hline & $\begin{array}{l}\text { Educación/asesoría, comunicación con el paciente, adherencia y } \\
\text { prevención de la automedicación }\end{array}$ & $49(54 \%)$ \\
\hline & Dispensación & $18(20 \%)$ \\
\hline & Farmacovigilancia & $4(5 \%)$ \\
\hline & Buenas prácticas de farmacia & $3(4 \%)$ \\
\hline \multirow{8}{*}{ Otros servicios y procesos } & Legislación farmacéutica & $2(3 \%)$ \\
\hline & Plantas medicinales y suplementos dietarios & $1(1 \%)$ \\
\hline & Prescripción por parte de los farmacéuticos & $1(1 \%)$ \\
\hline & Acceso a medicamentos & $1(1 \%)$ \\
\hline & Comités de las farmacias & $1(1 \%)$ \\
\hline & Ética de la profesión farmacéutica & $1(1 \%)$ \\
\hline & Prescripción de medicamentos & $1(1 \%)$ \\
\hline & Procesos técnicos y administrativos & $1(1 \%)$ \\
\hline
\end{tabular}




\section{Desenlaces principales evaluados (resulta- dos primarios)}

Los investigadores realizaron la medición de uno o varios desenlaces asociados a los servicios asistenciales desde las farmacias y en el personal farmacéutico, principalmente las competencias labo-rales, específicamente, conocimientos, habilidades y/o actitudes (prácticas, destrezas o conductas). En 78 (87\%) estudios se evaluaron, mínimo uno de estos tres componentes.

Competencias laborales: en 16 estudios se evaluaron los tres elementos establecidos como competencias laborales de acuerdo con la OMS (conjunto de los conocimientos, las habilidades y las actitudes -prácticas, destrezas o conductas- acorde con cada proceso o problema de salud intervenido). Algunos estudios evaluaron la combinación, mínimo de dos de estas competencias: en 13 los conocimientos y actitudes, en 13 los conocimientos y habilidades, y en 10 estudios las habilidades y actitudes. Por su parte, algunos estudios evaluaron una sola competencia: 9 los conocimientos, 7 las habilidades y 10 las actitudes. En 8 estudios se evaluó además, si el personal farmacéutico había percibido cambios en sus conocimientos, habilidades y/o actitudes. En 3 estudios se evaluó en el personal farmacéutico la intención de incorporar nuevas competencias en la práctica diaria. Además, en 12 estudios se evaluó la efectividad de la intervención de sus competencias en los pacientes y en 6 de estos estudios, se evaluó el suministro de la información que se facilitó en la intervención para que fuera entregada a los pacientes o utilizada por el personal farmacéutico en la práctica diaria.

Satisfacción: en 32 estudios (36\%) se evaluó la satisfacción de la intervención o el programa educativo implementado, en $12(13 \%)$ la satisfacción con el aprendizaje y en $12(13 \%)$ la utilidad de la intervención educativa.

Confianza: en 18 estudios (20\%), en el personal farmacéutico, se evaluaron los cambios en la confianza, principalmente en la realización de sus actividades, en sus habilidades y actitudes para evaluar las necesidades y el impacto de su aprendizaje, así como la disposición para el cambio. Además, en 17 (19\%) estudios se evaluó la aplicación de los elementos de la intervención en la práctica diaria.

Percepciones: en 5 estudios (6\%) se evaluaron las percepciones en el personal farmacéutico sobre el rol, la legitimización, la autoeficacia con respecto a las prácticas y la creencia de que tienen suficientes conocimientos, además de la motivación en sus funciones. Dos estudios evaluaron la oportunidad de trabajar en red con otros colegas.

Barreras y facilitadores: en 6 estudios (7\%) se analizaron las barreras y facilitadores del aprendizaje. Adicionalmente, un grupo de investigadores en sus dos estudios describieron que realizaron discusiones con el personal farmacéutico, buscando recolectar información sobre los alcances, patrones y factores que influenciaron en la participación de la intervención y la generación de planes de acción.

\section{Discusión}

Está revisión describió los programas e intervenciones educativas ofrecidas al personal farmacéutico para mejorar la prestación de los servicios asistenciales farmacéuticos, así como los métodos y estrategias utilizadas para evaluar la efectividad de estos programas. Los hallazgos de la revisión pueden ayudar a los sistemas de salud, asociaciones y agremiaciones del personal farmacéutico, como también a las instituciones educativas a desarrollar intervenciones de entrenamiento, programas de EC y de DPC de mayor calidad e impacto en las competencias laborales. Por ejemplo, en el contexto colombiano se ha planteado un estudio que busca establecer el impacto de un programa de educación continua para la utilización adecuada de medicamentos fiscalizados en la mejora de las competencias laborales del personal farmacéuticos de droguerías y farmacias-droguerías ${ }^{22}$.

Esta revisión no se centró en evaluar la efectividad de los programas e intervenciones o de los métodos utilizados; sin embargo, los resultados sugieren la necesidad de estandarizar las terminologías de los programas, técnicas y métodos de recolección de la información y resultados para evaluar la efectividad de los programas y las intervenciones educativas, y la comparación de los diferentes métodos y las estrategias utilizadas en diferentes estudios. Sobre la evaluación de los programas de EC, algunos autores han recomendado esta evaluación utilizando algún tipo de modelo, por ejemplo, evaluar en los sujetos intervenidos independientemente la satisfacción y la reacción, los resultados del aprendizaje, los cambios en el rendimiento y los comportamientos, y los resultados en el paciente o en su salud ${ }^{23}$. Algunos autores recomiendan la utilización de este tipo de modelos para comparar los diferentes programas e identificar las estrategias más efectivas ${ }^{13}$. 
En algunos estudios se ha demostrado que el aprendizaje autodirigido es más frecuente que los programas formales de educación continua ${ }^{6}$ y que la educación continua tradicional por sí sola, no facilita cambios en la práctica ${ }^{7,8}$. Internacionalmente, el personal farmacéutico elige varias formas de actualizar sus competencias principalmente, a través de congresos, seminarios, conferencias, cursos cortos y talleres en horarios extralaborales; algunos realizan programas de $\mathrm{EC}$ o de $\mathrm{DPC}^{12}$. Estos programas pueden desempeñar un papel vital en la expansión de la educación básica del personal farmacéutico y el fortalecimiento de las competencias laborales. Actualmente, algunos países están implementando programas de EC para farmacéuticos obligatorios para mejorar los servicios asistenciales $^{13}$.

Con relación a los términos y definiciones de los programas educativos evaluados, se evidenció que existen diferentes modos de definirlos, por ejemplo, curso de entrenamiento farmacéutico ${ }^{24}$, educación personalizada $^{25}$, entrenamiento e información continua ${ }^{26}$, entrenamiento y supervisión de apoyo ${ }^{27}$, programa de entrenamiento interprofesional ${ }^{28}$, intervención educacional ${ }^{29,30}$, intervención educativa ${ }^{31-35}$, red de investigación basada en la práctica ${ }^{36}$, programa de entrenamiento ${ }^{37-40}$, programa de entrenamiento comprensivo $^{41}$, o taller interactivo ${ }^{42}$. Adicionalmente, para los programas de EC, los términos utilizados fueron programa de aprendizaje combinado ${ }^{43}$, curso de desarrollo profesional ${ }^{44}$, educación farmacéutica continua $^{45,46}$, programa certificado $^{47}$, programa de aprendizaje $^{48}$, programa de educación profesional continuo $^{49}$, programa educativo ${ }^{50}$, programa de red de formación y comunicación ${ }^{51,52}$, o programa de entrenamiento del aprendizaje ${ }^{53}$. En dos estudios, el entrenamiento no tenía una definición concreta, sino que utilizaron un instrumento de medición validado mediante un marco de competencias o GLF (General Level Framework) $)^{54,55}$.

Por lo general, los estudios realizados en países de ingresos medios-altos utilizan los GLF como instrumento para evaluar el logro de los objetivos. En este sentido, es necesario destacar la importancia de este tipo de herramienta de desarrollo de desempeño, debido a que se basa en competencias laborales, proporciona una estructura para el desarrollo de los farmacéuticos en su capacidad profesional y facilita la retroalimentación y planificación del aprendizaje, basado en las necesidades y responsabilidad de un profesional con más experiencia ${ }^{56}$. En este contexto, esta revisión muestra que las competencias laborales son uno de los desenlaces más evaluados, además de otros aspectos como la satisfacción con la intervención y la confianza generada en ellos. Por su parte, se reconoce que los programas de EC y de DPC son estrategias adecuadas para actualizar competencias laborales ${ }^{57}$.

El conocimiento del personal farmacéutico es un requisito fundamental para la prestación de servicios de calidad, pero no siempre reflejan o están directamente relacionados con las habilidades y las actitudes en sus prácticas reales ${ }^{58}$. Sin embargo, la educación es la intervención más utilizada para mejorar las prácticas en el personal farmacéutico en todo el mundo ${ }^{59}$. En el personal farmacéutico, el cambio de conocimientos, comportamientos y prácticas puede ser un proceso lento, pero con probabilidad de lograr mejoras ${ }^{60}$. En este sentido, es posible mejorar las prácticas mediante la combinación de enfoques, métodos e intervenciones y además, la mayoría del personal farmacéutico está dispuesto a expresar sus necesidades de capacitación ${ }^{61}$.

Los programas de DPC y de EC pueden desempeñar un papel vital en la cobertura de la educación básica en farmacia y en la mejora de las competencias en la gestión de la farmacoterapia, particularmente en áreas con limitaciones durante los estudios de pregrado ${ }^{12}$. Se requiere de estos programas para garantizar que los profesionales estén actualizados y que brinden una atención óptima al paciente. Estos programas, principalmente los de DPC son autodirigidos y apoyan el mantenimiento del conocimiento, las habilidades y los comportamientos necesarios para una práctica efectiva ${ }^{11}$. Los conocimientos deben actualizarse regularmente para mantenerse al día con los cambios de $\mathrm{rol}^{62}$.

Esta revisión tiene algunas limitaciones, entre ellas la restricción del idioma en los criterios de inclusión, al igual que la revisión solo en PubMed/Medline; sin embargo, la inclusión de referencias bibliográficas, consideradas como relevantes, de los estudios revisados podría contrarrestar estas limitaciones.

\section{Conclusiones}

Se describen los programas y las intervenciones educativas ofrecidas al personal farmacéutico, principalmente para mejorar sus competencias laborales y la prestación de los servicios asistenciales farmacéuticos, además de los métodos utilizados para evaluar la efectividad de las intervenciones educativas. Se encontró que estos programas son más comunes en países desarrollados en los cuales se utilizan diferentes 
diseños metodológicos para describir y evaluar las intervenciones. Se observa que los principales desenlaces evaluados son los conocimientos, las habilidades y las actitudes (prácticas, destrezas o conductas) en el personal farmacéutico y que la presencialidad es la forma de entrega de la intervención más utilizada.

\section{Agradecimientos}

A la Universidad de Antioquia y a la Facultad de Ciencias Farmacéuticas y Alimentarias de la Universidad de Antioquia. Al Comité para el Desarrollo de la Investigación de la Universidad de Antioquia por la financiación del estudio a través de la convocatoria de sostenibilidad 2018-2019.

\section{Conflicto de interés}

Los autores declaran no tener ningún conflicto de intereses en el trabajo realizado.

\section{Rerencias}

1. Christensen DB, Farris KB. Pharmaceutical care in community pharmacies: Practice and research in the US. Ann Pharmacother. 2006; 40(7-8): 1400-1406. https://doi.org/10.1345/aph.1G545

2. Organización Mundial de la Salud OMS. Federación Internacional Farmacéutica FIP. Directrices Conjuntas FIP/OMS sobre Buenas Prácticas en Farmacia: Estándares para la calidad de los servicios farmacéuticos. 2011. https:/www. paho.org/hq/dmdocuments/2013/GPP-guidelinesFIP-publication-ES-2011.pdf

3. Vacca CP, Niño CY, Reveiz L. Restriction of antibiotic sales in pharmacies in Bogotá, Colombia: a descriptive study. Rev Panam Salud Publica. 2011; 30(6): 586-591. PMID: 22358407.

4. Ceballos M, Giraldo J, Giraldo J, Marín V, Amariles P. Caracterización de aspectos relacionados con la utilización de los medicamentos fiscalizados en droguerías y farmacias-droguerías de Medellín y el área metropolitana. Rev Univ Ind Santander Salud. 2018; 50(1): 27-36. doi: http://dx.doi. org/10.18273/revsal.v50n1-2018003

5. Alshammari TM, Alhindi SA, Alrashdi AM, Benmerzouga I, Aljofan M. Pharmacy Malpractice: The rate and prevalence of dispensing high-risk prescription-only medications at community pharmacies in Saudi Arabia. Saudi Pharm J. 2017; 25(5): 709-714. doi: 10.1016/j.jsps.2016.10.001

6. Schostak J, Davis M, Hanson J, Schostak J, Brown T, Driscoll P, et al. Effectiveness of continuing professional development project: A summary of findings. Med Teach. 2010; 32(7): 586-592. doi: 10.3109/0142159X.2010.489129

7. McConnell KJ, Delate T, Newlon CL. Impact of continuing professional development versus traditional continuing pharmacy education on learning behaviors. J Am Pharm Assoc. 2012; 52(6): 742-752. doi: https://doi.org/10.1331/ JAPhA.2012.11080

8. Davis D, O'Brien MAT, Freemantle N, Wolf FM, Mazmanian P, Taylor-Vaisey A. Impact of formal continuing medical education: Do conferences, workshops, rounds, and other traditional continuing education activities change physician behavior or health care outcomes? JAMA. 1999; 282(9): 867-874. doi: 10.1001/jama.282.9.867

9. Organización Panamericana de la Salud PAHO. Propuesta de Plan Básico de Educación Farmacéutica y Competencias del Farmacéutico para la práctica profesional Conferencia Panamericana de Educación Farmacéutica (CPEF). 2014. http://www.observatoriorh.org/ sites/default/files/webfiles/fulltext/2014/ix cpef/ PlanBasico.pdf

10. Organización Mundial de la Salud OMS. Federación Internacional Farmacéutica FIP. Competencias del farmacéutico para desarrollar los servicios farmacéuticos (SF) basados en Atención Primaria de Salud (APS) y las Buenas Prácticas en Farmacia (BPF). Grupo Técnico para el desarrollo de competencias para los Servicios Farmacéuticos (GT-CSF). 2012. https://www.paho.org/es/ documentos/competencias-farmaceutico-paradesarrollar-servicios-farmaceuticos-sf-basadosatencion

11. Rouse MJ. Continuing professional development in pharmacy. J Am Pharm Assoc. 2004; 44(4): 517-520. doi: 10.1331/1544345041475634

12. Federación Internacional Farmacéutica FIP. International Pharmaceutical Federation. Continuing professional development/continuing education in pharmacy: Global repor. 2014. https:// www.fip.org/file/1407

13. Obreli-Neto PR, Marques dos Reis T, Guidoni CM, Girotto E, Guerra ML, de Oliveira Baldoni A, et al. A Systematic review of the effects of continuing education programs on providing clinical community pharmacy services. Am J Pharm Educ. 2016; 80(5): 88. doi: 10.5688/ajpe80588

14. Ceballos JM, Amariles P. Needs and opportunities for outpatient pharmacies in colombia: The case of continuing education programs for pharmacists. Vitae. 2017; 24(1). doi: http://dx.doi.org/10.17533/ udea.vitae.v24n1a01 
15. Farrell B, Jennings B, Ward N, Marks PZ, Kennie N, Dolovich L, et al. Evaluation of a pilot e-learning primary health care skills training program for pharmacists. Curr Pharm Teach Learn. 2013; 5(6): 580-592. doi: https://doi.org/10.1016/j. cptl.2013.07.005

16. Farrell B, Ward N, Jennings B, Jones C, Jorgenson $\mathrm{D}$, Gubbels-Smith A, et al. Participation in online continuing education. Int J Pharm Pract. 2016; 24(1): 60-71. doi: http://doi.wiley.com/10.1111/ ijpp. 12202

17. Buxton EC, De Muth JE. Pharmacists' perceptions of a live continuing education program comparing distance learning versus local learning. Res Social Adm Pharm. 2013; 9(2): 230-235. doi: https://doi. org/10.1016/j.sapharm.2012.05.003

18. Hudmon KS, Hoch MA, Vitale FM, Wahl KR, Corelli RL, Moor C de. Tobacco cessation education for pharmacists: Face-to-face presentations versus live webinars. J Am Pharm Assoc. 2014; 54(1): 4244. doi: 10.1331/JAPhA.2014.13001

19. Hughes CA, Schindel TJ. Evaluation of a professional development course for pharmacists on laboratory values: can practice change? Int $\mathrm{J}$ Pharm Pract. 2010; 18(3): 174-179.

20. Javadi M, Kargar A, Gholami K, Hadjibabaie M, Rashidian A, Torkamandi H, et al. Didactic lecture versus interactive workshop for continuing pharmacy education on reproductive health: A Randomized Controlled Trial. Eval Health Prof. 2015; 38(3): 404-418. doi: https://doi. org/10.1177/0163278713513949

21. Sarayani A, Rashidian A, Gholami K, Torkamandi $\mathrm{H}$, Javadi M. Efficacy of continuing education in improving pharmacists' competencies for providing weight management service: three-arm randomized controlled trial. J Contin Educ Health Prof. 2012; 32(3): 163-73. doi: 10.1002/chp. 21141

22. Ceballos M, Salazar-Ospina A, Sabater-Hernández D, Amariles P. Evaluation of the effects of a drug with fiscalized substance dispensation, health education, and pharmacovigilance continuing education program in Colombia drugstores and drugstores/pharmacies: study protocol of a multicenter, cluster-randomized controll. Trials. 2020; 21(545): doi: https://doi.org/10.1186/ s13063-020-04481-1

23. Donald Kirkpatrick JK. Evaluating Training Programs: The Four Levels. 2008.

24. Dhital R, Whittlesea CM, Milligan P, Khan NS, Norman IJ. The impact of training and delivering alcohol brief intervention on the knowledge and attitudes of community pharmacists: A before and after study. Drug Alcohol Rev 2013; 32(2): 147156. doi: 10.1111/j.1465-3362.2012.00513.x

25. Meštrović A, Staničić Z, Hadžiabdić MO, Mucalo I, Bates I, Duggan C, et al. Individualized education and competency development of Croatian community pharmacists using the general level framework. Am J Pharm Educ. 2012; 76(2): 23; doi: https://doi.org/10.5688/ajpe76223

26. Terblanche A, Meyer JC, Godman B, Summers RS. Impact of a pharmacist-driven pharmacovigilance system in a secondary hospital in the Gauteng Province of South Africa. Hosp Pract. 2018; 46(4): 221-228. doi: https://doi.org/10.1080/21548331.2 018.1510708

27. Minh PD, Huong DTM, Byrkit R, Murray M. Strengthening pharmacy practice in vietnam: findings of a training intervention study. Trop Med Int Health. 2013; 18(4): 426-434. doi: 10.1111/ tmi.12062

28. Bajorek B V, Lemay KS, Magin PJ, Roberts C, Krass I, Armour CL. Preparing pharmacists to deliver a targeted service in hypertension management: evaluation of an interprofessional training program. BMC Med Educ. 2015; 15(1): 157. doi: https://doi.org/10.1111/tmi.12062

29. Pham DM, Byrkit M, Pham H Van, Pham T, Nguyen CT. Improving pharmacy staff knowledge and practice on childhood diarrhea management in Vietnam: Are educational interventions effective? PLoS One. 2013; 8(10):e74882. doi: 10.1371/ journal.pone.0074882

30. Ngwerume K, Watson M, Bond C, Blenkinsopp A. An evaluation of an intervention designed to improve the evidence-based supply of non-prescription medicines from community pharmacies. Int J Pharm Pract. 2015; 23(2): 102110. doi: https://doi.org/10.1111/ijpp.12118

31. Chowdhury F, Sturm-Ramirez K, Mamun A $\mathrm{Al}$, Iuliano $\mathrm{AD}$, Chisti MJ, Ahmed $\mathrm{M}$, et al. Effectiveness of an educational intervention to improve antibiotic dispensing practices for acute respiratory illness among drug sellers in pharmacies, a pilot study in Bangladesh. BMC Health Serv Res. 2018; 18(1): 676. doi: 10.1186/ s12913-018-3486-y

32. Jha N, Rathore DS, Shankar PR, Bhandary S, Pandit RB, Gyawali S, et al. Effect of an educational intervention on knowledge and attitude regarding pharmacovigilance and consumer pharmacovigilance among community pharmacists in Lalitpur district, Nepal. BMC Res Notes. 2017; 10(1): 4. doi: https://doi.org/10.1186/ s13104-016-2343-5 
33. Hussain A, Ibrahim MI, Malik M. Impact of educational intervention on knowledge of dispensers working at community pharmacies in Pakistan. Pharm Pract (Granada). 2013; 11(3): 144148. doi: 10.4321/s1886-36552013000300004

34. O’Neal KS, Murray KA, Skomo ML, Carter SM, McConaha J. Validation of a survey tool assessing effectiveness of an educational intervention on the caring behaviors and referral activities of community pharmacists for migraineurs. Res Soc Adm Pharm. 2015; 11(3): 352-363. doi: 10.1016/j. sapharm.2014.08.012

35. Zillich AJ, Corelli RL, Zbikowski SM, Magnusson LB, Fenlon CM, Prokhorov AV, et al. A randomized trial evaluating 2 approaches for promoting pharmacy-based referrals to the tobacco quitline: Methods and baseline findings. Res Soc Adm Pharm. 2013; 9(1): 27-36. doi: https://doi. org/10.1016/j.sapharm.2012.03.001

36. Chartrand M, Lalonde L, Cantin A, Lahaie A, Odobasic B, Tremblay MP, et al. Anticoagulation management services in community pharmacy: Feasibility of implementing a quality improvement programme through a practice-based research network. J Clin Pharm Ther. 2018; 43(6): 877-887. doi: $10.1111 /$ jcpt. 12745

37. Thomas T, Passfield L, Coulton S, Crone D. Effectiveness of a tailored training programme in behaviour change counselling for community pharmacists: A pilot study. Patient Educ Couns. 2016; 99(1): 132-138. doi: https://doi. org/10.1016/j.pec.2015.08.004

38. Gheewala PA, Peterson GM, Zaidi STR, Bereznicki L, Jose MD, Castelino RL. A webbased training program to support chronic kidney disease screening by community pharmacists. Int J Clin Pharm. 2016; 38(5): 1080-1086. doi: https:// doi.org/10.1007/s11096-016-0330-5

39. O'Reilly CL, Wong E, Chen TF. A feasibility study of community pharmacists performing depression screening services. Res Soc Adm Pharm. 2015; 11(3): 364-381. doi: 10.1016/j. sapharm.2014.08.013

40. Longman C, Lintzeris N, Temple-Smith $\mathrm{M}$, Gilchrist G. Methadone and buprenorphine prescribing patterns of Victorian general practitioners: their first 5 years after authorisation. Drug Alcohol Rev. 2011; 30(4): 355-359. doi: 10.1111/j.1465-3362.2010.00212.x
41. Palmer E, Hart S, Freeman PR. Development and delivery of a pharmacist training program to increase naloxone access in Kentucky. J Am Pharm Assoc. 2017; 57(2S): S118-122. doi: https://doi. org/10.1016/j.japh.2016.12.071

42. Guirguis LM, Lee S, Sanghera R. Impact of an interactive workshop on community pharmacists' beliefs toward patient care. Int J Clin Pharm. 2012; 34(3): 460-467. doi: 10.1007/s11096-012-9630-6

43. Laven A, Deters MA, Rose O, Schwender H, Smaranda A, Waltering I, et al. PharmAdhere: training German community pharmacists with Objective Structured Clinical Examinations. Int J Clin Pharm. 2018; 40(5): 1317-1327. doi: https:// doi.org/10.1007/s11096-018-0710-0

44. Bungard TJ, Schindel TJ, Garg S, Brocklebank C. Evaluation of a multi staged professional development course for practising pharmacists in anticoagulation management. Int $\mathrm{J}$ Pharm Pract. 2012; 20(2): 107-117. doi: https://doi.org/10.1111/ j.2042-7174.2011.00171.x

45. Fjortoft N. The effectiveness of commitment to change statements on improving practice behaviors following continuing pharmacy education. Am J Pharm Educ. 2007; 71(6): 112. doi: 10.5688/ aj7106112

46. Diamantouros A, Marchesano R, Geerts WH, Pennefather $\mathrm{P}$, Zwarenstein M, Austin Z. Development and evaluation of a continuing pharmacy education (CPE) program in thrombosis management. Curr Pharm Teach Learn. 2017; 9(5): 911-917. doi: https://doi.org/10.1016/j. cptl.2017.05.008

47. Kirk JK, Edwards R, Brewer A, Miller C, Bray B, Groce JB. Overview of a pharmacist anticoagulation certificate program. Curr Pharm Teach Learn. 2017; 9(4): 707-712. doi: https://doi. org/10.1016/j.cptl.2017.03.015

48. Moczygemba LR, Pierce AL, Dang A, Emberley P, Czar MJ, Matzke GR. The ADAPT online education program: A tool for practicing pharmacists delivering patient-centered care. J Am Pharm Assoc. 2017; 57(5): 601-617. doi: https:// doi.org/10.1016/j.japh.2017.05.007

49. Martin BA, Bruskiewitz RH, Chewning BA. Effect of a tobacco cessation continuing professional education program on pharmacists' confidence, skills, and practice-change behaviors. J Am Pharm Assoc. 2010; 50(1): 9-18a. doi: 10.1331/ JAPhA.2010.09034 
50. Stafford L, Peterson GM, Bereznicki LR, Jackson SL, van Tienen EC. Training Australian pharmacists for participation in a collaborative, home-based post-discharge warfarin management service. Pharm World Sci. 2010; 32(5): 637-642. doi: https://doi.org/10.1007/s11096-010-9416-7

51. Legris M-È, Séguin NC, Desforges K, Sauvé P, Lord A, Bell R, et al. Pharmacist Web-based training program on medication use in chronic kidney disease patients: impact on knowledge, skills, and satisfaction. J Contin Educ Health Prof. 2011; 31(3): 140-150. doi: 10.1002/chp.20119

52. Lalonde L, Quintana-Bárcena P, Lord A, Bell R, Clément V, Daigneault AM, et al. Community pharmacist training-and-communication network and drug-related problems in patients with CKD: A multicenter, cluster-randomized, controlled trial. Am J Kidney Dis. 2017; 70(3): 386-396. doi: https://doi.org/10.1053/j.ajkd.2017.05.008

53. Lee SWH, Mak VSL. Train-the-trainer program on cardiovascular health for community pharmacists in Malaysia. Int J Clin Pharm. 2017; 39(6): 11661170. doi: 10.1007/s11096-017-0540-5

54. Svetlana S, Ivana T, Tatjana C, Duskana K, Ian B. Evaluation of competences at the community pharmacy settings. Indian $\mathrm{J}$ Pharm Educ Res. 2014; 48(4): 22-30. doi: 10.5530/ijper.48.4.4

55. Coombes I, Avent M, Cardiff L, Bettenay K, Coombes J, Whitfield K, et al. Improvement in Pharmacist's performance facilitated by an adapted competency-based general level framework. J Pharm Pract Res. 2010; 40(2): 111-118. doi: https:// doi.org/10.1002/j.2055-2335.2010.tb00517.x

56. Rutter V, Wong C, Coombes I, Cardiff L, Duggan $\mathrm{C}$, Yee ML, et al. Use of a general level framework to facilitate performance improvement in hospital pharmacists in Singapore. Am J Pharm Educ. 2012; 76(6): 107. doi: https://doi.org/10.5688/ ajpe 766107

57. Micallef, Kayyali. A Systematic Review of Models Used and Preferences for Continuing Education and Continuin1. Micallef, Kayyali. A systematic review of models used and preferences for continuing education and continuing professional development of pharmacists. Pharmacy. 2019; 7(4): 154. doi: 10.3390/pharmacy7040154

58. Chalker J, Chuc NTK, Falkenberg T, Do NT, Tomson G. STD management by private pharmacies in Hanoi: Practice and knowledge of drug sellers. Sex Transm Infect. 2000; 76(4): 299302. doi: 10.1136/sti.76.4.299
59. Smith F. The quality of private pharmacy services in low and middle-income countries: A systematic review. Pharm World Sci; 2009; 31(3): 351-361. doi: 10.1007/s11096-009-9294-z

60. Laing R, Hogerzeil H, Ross-Degnan D. Ten recommendations to improve use of medicines in developing countries. Health Policy Plan. 2001; 16(1): 13-20. doi: 10.1093/heapol/16.1.13

61. Hussain A, Ibrahim M, Izham M. Qualification, knowledge and experience of dispensers working at community pharmacies in Pakistan. Pharmacy Pract (Granada). 2011; 9(2): 93-100.

62. Toklu HZ, Hussain A. The changing face of pharmacy practice and the need for a new model of pharmacy education. J Young Pharm. 2013; 5(2): 38-40. doi: 10.1016/j.jyp.2012.09.001

63. Adepu R, Shariff A. Development, validation and implementation of continuous professional development programmes for community pharmacists. Indian J Pharm Sci. 2010; 72(5): 557. doi: 10.4103/0250-474X.78520

64. Augustine J, Warholak TL, Hines LE, Sun D, Brown M, Hurwitz J, et al. Ability and use of comparative effectiveness research by P\&amp; $\mathrm{T}$ Committee Members and Support Staff: A 1-Year Follow-up. J Manag Care Spec Pharm. 2016; 22(6): 618-625. doi: https://doi.org/10.18553/ jmcp.2016.22.6.618

65. Batra P, Aquilino ML, Farris KB. Pharmacy staff perceptions and self-reported behaviors related to providing contraceptive information and counseling. J Am Pharm Assoc. 2015; 55(5): 481-487. doi: https://doi.org/10.1331/ JAPhA.2015.15037

66. Buxton EC, Burns EC, De Muth JE. Professional development webinars for pharmacists. Am J Pharm Educ. 2012; 76(8): 155. doi: 10.5688/ ajpe 768155

67. Buxton EC. Pharmacists' Perception of synchronous versus asynchronous distance learning for continuing education programs. Am J Pharm Educ. 2014; 78(1): 8. doi: 10.5688/ ajpe 7818

68. Charpentier MM, Orr KK, Taveira TH. Improving pharmacist knowledge of oral chemotherapy in the community. Ann Pharmacother. 2012; 46(9): 1205-1211. doi: 10.1345/aph.1R056

69. Chiang YC, Lee CN, Lin YM, Yen YH, Chen HY. Impact of a continuing education program on pharmacists' knowledge and attitudes toward asthma patient care. Med Princ Pract. 2010; 19(4): 305-311. doi: 10.1159/000312718 
70. Condren ME, Desselle SP. The fate of pediatric prescriptions in community pharmacies. J Patient Saf. 2015; 11(2): 79-88. doi: 10.1097/ PTS.0b013e3182948a7d

71. Coombes I, Avent M, Cardiff L, Bettenay K, Coombes $\mathrm{J}$, Whitfield $\mathrm{K}$, et al. Improvement in pharmacist's performance facilitated by an adapted competency-based general level framework. J Pharm Pract Res. 2010; 40(2):111-118. doi: https:// doi.org/10.1002/j.2055-2335.2010.tb00517.x

72. Donyai P, Alexander AM. Training on the use of a bespoke continuing professional development framework improves the quality of CPD records. Int J Clin Pharm. 2015; 37(6): 1250-1257. doi: https://doi.org/10.1007/s11096-015-0202-4

73. Dopp AL, Moulton JR, Rouse MJ, Trewet CB. A five-state continuing professional development pilot program for practicing pharmacists. Am J Pharm Educ. 2010; 74(2): 28. doi: 10.5688/ aj740228

74. Dualde E, Faus MJ, Santonja FJ, FernandezLlimos F. Effectiveness of a videoconference training course on implementing pharmacy services. Pharm World Sci. 2009; 31(6): 638-642. doi: 10.1007/s11096-009-9317-9

75. Dualde E, Font G, Santonja FJ, Faus MJ. Impact of pharmacists' participation in a pharmacotherapy follow-up program. Am J Pharm Educ. 2012; 76(2): 34. doi: 10.5688/ajpe76234

76. Elaro A, Shah S, Pomare LN, Armour CL, BosnicAnticevich S. PACE: Pharmacists use the power of communication in paediatric asthma. Int $\mathrm{J}$ Clin Pharm. 2014; 36(5): 976-985. doi: 10.1007/ s11096-014-9984-Z

77. Feletto E, Lui GWY, Armour C, Saini B. Practice change in community pharmacy: using changemanagement principles when implementing a pharmacy asthma management service in NSW, Australia. Int J Pharm Pract. 2013; 21(1): 28-37. doi: https://doi.org/10.1111/j.20427174.2012.00225.x

78. Fitzgerald N, Watson H, McCaig D, Stewart D. Developing and evaluating training for community pharmacists to deliver interventions on alcohol issues. Pharm World Sci. 2009; 31(2): 149-153. doi: https://doi.org/10.1007/s11096-009-9284-1

79. Formea CM, Nicholson WT, McCullough KB, Berg KD, Berg ML, Cunningham JL, et al. Development and evaluation of a pharmacogenomics educational program for pharmacists. Am J Pharm Educ. 2013; 77(1): 10. doi: https://doi.org/10.5688/ajpe77110
80. García PJ, Carcamo CP, Garnett GP, Campos PE, Holmes KK. Improved STD syndromemanagement by a network of clinicians and pharmacy workers in Peru: The PREVEN Network. Vermund SH, editor. PLoS One. 2012; 7(10): e47750. doi: https://doi.org/10.1371/journal.pone.0047750

81. Grzeskowiak LE, Thomas AE, To J, Reeve E, Phillips AJ. Enhancing continuing education activities using audience response systems: a single-blind controlled trial. J Contin Educ Health Prof. 2015; 35(1): 38-45. doi: 10.1002/chp.21269

82. Hussainy SY, Marriott JL, Beattie J, Nation RL, Dooley MJ. A palliative cancer care flexible education program for Australian community pharmacists. Am J Pharm Educ. 2010; 74(2): 24. doi: 10.5688/aj740224

83. Kjome RLS, Nerhus K, Sandberg S. Implementation of a method for glucose measurements in community pharmacies. Int J Pharm Pract. 2010; 18(1): 13-19. doi: 10.1211/ijpp.18.01.0004

84. Kouladjian L, Chen TF, Gnjidic D, Hilmer SN. Education and assessment of pharmacists on the use of the drug burden index in older adults using a continuing professional development education method. Am J Pharm Educ. 2016; 80(4): 63 doi: 10.5688/ajpe 80463

85. Kristina SA, Thavorncharoensap M, Pongcharoensuk P, Prabandari YS. Impact of smoking cessation training for community pharmacists in Indonesia. Asian Pac J Cancer Prev. 2015; 16(8): 3319-3323. doi: 10.7314/ apjcp.2015.16.8.3319

86. Kuo GM, Lee KC, Ma JD. Implementation and outcomes of a live continuing education program on pharmacogenomics. Pharmacogenomics. 2013; 14(8): 885-895. doi: 10.2217/pgs. 13.77

87. Laaksonen R, Bates I, Duggan C. Training, clinical medication review performance and self-assessed competence: Investigating influences. Pharmacy Education. 2007; 7(3): 257-265.

88. Laaksonen R, Duggan C, Bates I. Performance of community pharmacists in providing clinical medication reviews. Ann Pharmacother. 2010; 44(7-8): 1181-1190. doi: https://doi.org/10.1345/ aph.1M719

89. Lalonde L, Normandeau M, Lamarre D, Lord A, Berbiche D, Corneille L, et al. Evaluation of a training and communication-network nephrology program for community pharmacists. Pharm World Sci. 2008; 30(6): 924-933. doi: $10.1007 /$ s11096-008-9253-0 
90. Liekens S, Smits T, Laekeman G, Foulon V. A depression training session with consumer educators to reduce stigmatizing views and improve pharmacists' depression care attitudes and practices. Am J Pharm Educ. 2013; 77(6): 120. doi: 10.5688/ajpe 776120

91. Liekens S, Vandael E, Roter D, Larson S, Smits $\mathrm{T}$, Laekeman $\mathrm{G}$, et al. Impact of training on pharmacists' counseling of patients starting antidepressant therapy. Patient Educ Couns. 2014; 94(1): 110-115. doi: https://doi.org/10.1016/j. pec.2013.09.023

92. Martin BA, Chewning BA. Evaluating pharmacists' ability to counsel on tobacco cessation using two standardized patient scenarios. Patient Educ Couns. 2011; 83(3): 319-324. doi: https://doi. org/10.1016/j.pec.2010.12.010

93. McConnell KJ, Newlon CL, Delate T. The impact of continuing professional development versus traditional continuing pharmacy education on pharmacy practice. Ann Pharmacother. 2010; 44(10): 1585-1595. doi: https://doi.org/10.1345/ aph.1P161

94. McConnell KJ, Delate T, Newlon CL. The sustainability of improvements from continuing professional development in pharmacy practice and learning behaviors. Am J Pharm Educ. 2015; 79(3): 36. doi: 10.5688/ajpe79336

95. Melo DO de, Molino CGR da C, Ribeiro E, Romano-Lieber NS. Capacitação e intervenções de técnicos de farmácia na dispensação de medicamentos em Atenção Primária à Saúde. Cien Saude Colet. 2017; 22(1): 261-268. doi: 10.1590/1413-81232017221.1612201

96. Obreli-Neto PR, Guidoni CM, Baldoni AO, Marusic S, Castedalli G, Lahoud YM, et al. Evaluation of the effectiveness of an Internetbased continuing education program on pharmacybased minor ailment management: a randomized controlled clinical trial. Brazilian J Pharm Sci. 2016; 52(1): 15-26. doi: https://doi.org/10.1590/ S1984-82502016000100003

97. O’Neal KS, Crosby KM, Miller MJ, Murray KA, Condren ME. Assessing health literacy practices in a community pharmacy environment: experiences using the AHRQ Pharmacy Health Literacy Assessment Tool. Res Social Adm Pharm. 2013; 9(5): 564-596. doi: https://doi.org/10.1016/j. sapharm.2012.09.005
98. Rouleau R, Beauchesne MF, Laurier C. Impact of a continuing education program on community pharmacists' interventions and asthma medication use: a pilot study. Ann Pharmacother. 2007; 41(4): 574-580. doi: https://doi.org/10.1345/aph.1H606

99. Sadler S, Rodgers S, Howard R, Morris CJ, Avery AJ, PINCER Triallists. Training pharmacists to deliver a complex information technology intervention (PINCER) using the principles of educational outreach and root cause analysis. Int J Pharm Pract. 2014; 22(1): 47-58. doi: https://doi. org/10.1111/ijpp.12032

100. Saw PS, Chuah LH, Lee SWH. A practical approach toward teaching ethics to community pharmacists. Int J Clin Pharm. 2018; 40(5): 11311136. doi: 10.1007/s11096-018-0707-8

101. Saylor MS, Blanchette LM, Smith MB, Cambron $\mathrm{K}$, Andricopulos $\mathrm{K}$, Brown MJ. Initiation and preliminary evaluation of an oncology pharmacy training course for staff pharmacists. J Oncol Pharm Pract. 2016; 22(4): 611-617. doi: https:// doi.org/10.1177/1078155215599834

102. Schafheutle EI, Smith C, Cutts C, Higginson PJ, Noyce PR. Pharmacy technicians' views of learning and practice implementation. Int J Pharm Pract. 2012; 20(3): 141-147. doi: https://doi. org/10.1111/j.2042-7174.2012.00186.x

103. Sidhu S, Gorman SK, Slavik RS, Ramsey T, Bruchet N, Murray S. Positive and negative impacts of a continuing professional development intervention on pharmacist practice: A balanced measure evaluation. J Contin Educ Health Prof. 2017; 37(4): 215-22. doi: 10.1097/CEH.0000000000000166

104. Smith SD, Lee A, Blaszczynski A, Fischer G. Pharmacists' knowledge about use of topical corticosteroids in atopic dermatitis: Pre and post continuing professional development education. Australas J Dermatol. 2016; 57(3): 199-204. doi: 10.1111/ajd.12339

105. Sweet B V, Welage LS, Johnston JP. Effect of a Web-based continuing-education program on pharmacist learning. Am J Health Syst Pharm. 2009; 66(21): 1902-1903. doi: https://doi. org/10.2146/ajhp080658

106. Temple ME, Jakubecz MA, Link NA. Implementation of a training program to improve pharmacy services for high-risk neonatal and maternal populations. Am J Heal Pharm. 2013; 70(2): 144-149. doi: 10.2146/ajhp110681 
107. Tofade1 T, Foushee L, Chou S, Caiola SM, Eckel S. Continuing professional development training program among pharmacist preceptors and nonpreceptors. J Am Pharm Assoc. 2010; 50(6): 730-735. doi: 10.1331/JAPhA.2010.09150

108. Tofade TS, Foushee LL, Chou SY, Eckel SF, Caiola SM. Evaluation of a condensed training program to introduce the process of continuing professional development. J Pharm Pract. 2010; 23(6): 560-569. doi: 10.1177/0897190010375852

109. Trewet CB, Fjortoft N. Evaluation of the impact of a continuing professional development worksheet on sustained learning and implementing change after a continuing pharmacy education activity. Res Soc Adm Pharm. 2013; 9(2): 215-221. doi: 10.1016/j.sapharm.2012.06.002

110. Wahl KR, Woolf BL, Hoch MA, Zillich AJ, Hudmon KS. Promoting pharmacy-based referrals to the tobacco quitline: a pilot study of academic detailing administered by pharmacy students. J Pharm Pract. 2015; 28(2): 162-165. doi: https:// doi.org/10.1177/0897190013515711

111. Walters C, Raymont A, Galea S, Wheeler A. Evaluation of online training for the provision of opioid substitution treatment by community pharmacists in New Zealand. Drug Alcohol Rev. 2012; 31(7): 903-910. doi: 10.1111/j.14653362.2012.00459.x

112. Wheeler A, Fowler J, Hattingh L. Using an intervention mapping framework to develop an online mental health continuing education program for pharmacy staff. J Contin Educ Health Prof. 2013; 33(4): 258-266. doi: 10.1002/chp.21198

113. Wilbur K, Shabana S, Maraghi F, ElMubark A, Kheir N. An evaluation of the translation of continuing education into diabetes public health care by pharmacists. Int J Clin Pharm. 2017; 39(4): 774-782. doi: https://doi.org/10.1007/s11096-0170477-8

114. Zembles T. An inservice program on pharmacogenetics to individualize drug therapy. Am J Pharm Educ. 2010; 74(1): 10. doi: 10.5688/ aj740110 